\title{
Multiple Time-Varying Formation of Networked Heterogeneous Robotic Systems via Estimator-Based Hierarchical Cooperative Algorithms
}

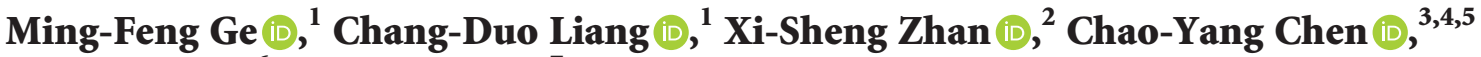 \\ Guanghui $X u{ }^{6}$ and Jie Chen $\mathbb{1}^{7}$ \\ ${ }^{1}$ School of Mechanical Engineering and Electronic Information, China University of Geosciences, Wuhan 430074, China \\ ${ }^{2}$ Department of Control Science and Engineering, Hubei Normal University, Huangshi 435002, China \\ ${ }^{3}$ School of Information and Electrical Engineering, Hunan University of Science and Technology, Xiangtan 411201, China \\ ${ }^{4}$ Center for Polymer Studies, Boston University, Boston, MA 02215, USA \\ ${ }^{5}$ Department of Physics, Boston University, Boston, MA 02215, USA \\ ${ }^{6}$ School of Electrical and Electronic Engineering, Hubei University of Technology, Wuhan 430068, China \\ ${ }^{7}$ School of Science, Hubei University of Technology, Wuhan 430068, China \\ Correspondence should be addressed to Chao-Yang Chen; ouzk@163.com
}

Received 23 June 2019; Revised 10 December 2019; Accepted 21 January 2020; Published 14 February 2020

Academic Editor: Hassan Zargarzadeh

Copyright (C) 2020 Ming-Feng Ge et al. This is an open access article distributed under the Creative Commons Attribution License, which permits unrestricted use, distribution, and reproduction in any medium, provided the original work is properly cited.

\begin{abstract}
This paper investigates both the time-varying formation and multiple time-varying formation tracking problems of networked heterogeneous robotic systems (NHRSs) with parameter uncertainties and external disturbances in the task space. Each robot inside can be either redundant or nonredundant. Several novel estimator-based hierarchical cooperative (EBHC) algorithms are designed to achieve both the tracking task and the possible preset subtasks for redundant robots. Besides, the designed estimator algorithms guarantee that each robot can obtain the accurate information of their corresponding leaders. By employing Lyapunov stability and input-to-state stability, sufficient conditions on the asymptotic stability of the error closed-loop system are derived. Finally, two simulation examples are presented to verify the effectiveness of the proposed algorithms.
\end{abstract}

\section{Introduction}

In the past decades, the researches on distributed cooperative control of multiagent systems have attracted increasing attentions for its superiority over the traditional centralized control, such as stronger robustness, less energy consumption, and more flexibility [1], which thus has brought out a series of related topics, including consensus [2-4], distributed optimization in smart grids [5], consensus tracking [6-8], optimal tracking [9-13], bipartite consensus/tracking [14, 15], formation [16-19], formationcontainment [20-22], synchronization [23-26], and flocking [27]. As a special case of the multiagent system, Euler-Lagrange dynamics are more applicative in many practical rigid structure modeling, especially the networked robotic system (NRS), which has gained increasing popularity due to its extensive potential applications, including industrial manufacture, search missions, and rescue missions [28].

Therefore, more and more researchers embark on the cooperative control of NRSs [3, 6, 29]. However, the aforementioned literature studies mainly focused on the joint-space control of NRSs. In real-world applications, the task-space algorithms are indeed more practical due to the consideration of the kinematics analysis, which thus leads to a few researches studying the task-space control of NRSs $[30,31]$. It noteworthy that the heterogeneity of NRSs has not been taken into account in the above researches. Besides, 
when performing tasks, redundant robots are generally more functional than the nonredundant robots for their extra degrees of freedom (DOFs) and usually utilized to perform some corresponding subtasks [32]. Thus, in a system and an application view, it is of great significance to investigate the task-space cooperative control of networked heterogeneous robotic systems (NHRSs).

With the development of consensus theory, there is a tendency for researchers to embark on the studies of formation problems. To be specific, Dong et al. have studied the time-varying formation tracking problem of multiagent systems under switching topologies in [18]. The time-invariant formation problems of multiagent systems with time delays have been solved in [33]. A distributed position estimation method is employed in [34] to realize the formation of multiagent systems. Ge et al. [35] have proposed a finitetime algorithm to achieve a switching type of time-varying formation for NRSs in the joint space. It should be pointed out that most of the aforementioned works focused on the time-invariant or time-varying formation in the case of a single leader, namely, all agents inside form a single formation. However, in many practical circumstances, the main results of the above literature studies cannot be directly applied if the networked systems are required to execute multiple tasks concurrently. A few works centralized on multiple tracking $[7,36,37]$, multiple formation $[16,17]$, and group time-varying formation [19] are thus activated. To the best of our knowledge, the time-varying formation tracking problem of NHRSs in the task space remains unsolved, let alone the multiple time-varying formation tracking problem.

On the contrary, in the practical robotic application, inexact physical parameters and external disturbances are ineluctable and usually have negative impacts on the performance of the NHRS. It thus becomes extremely challenging to study the time-varying formation tracking problem of NHRSs in the task space with parameter uncertainties and external disturbances.

Based on the above discussions, this paper investigates both the time-varying formation and multiple time-varying formation problems of NHRSs in the task space with parameter uncertainties and external disturbances. The main contributions of this paper can be summed up threefold: (1) The time-varying formation and multiple time-varying formation tracking problems of NHRSs in the task space are firstly studied and successfully addressed by designing several novel estimator-based hierarchical cooperative (EBHC) algorithms. (2) The proposed finite-time distributed estimator algorithm can guarantee the accurate estimation of the states of the virtual leaders, namely, the availability of leaders' information for each robot is assured. (3) Without interfering with the time-varying formation tracking task, the possible preset subtasks for redundant robots can also be accomplished.

Notations: let $\mathbb{R}^{n}$ equal the $n$-dimensional Euclidean space; especially, $\mathbb{R}$ represents the real number field. The Kronecker product is denoted by $\otimes . I_{m}$ is the $m$-order identity matrix, and $1_{m}\left(0_{m}\right)$ symbolizes an $m$-dimensional column vector with all its elements being $1(0) . \mathscr{L}_{2}$ and $\mathscr{L}_{\infty}$ denote the $\mathscr{L}_{2}$ space and $\mathscr{L}_{\infty}$ space. $\|\cdot\|$ and sup $\|\cdot\|$ denote the Euclidean norm and its supremum, respectively. For given $\ell>0$ and $\mathscr{I}=\left[\mathscr{I}_{1}, \mathscr{I}_{2}, \ldots, \mathscr{I}_{n}\right]^{T}$, one has $\operatorname{sig}(\mathscr{I})^{\ell}=$ $\left[\operatorname{sgn}\left(\mathscr{I}_{1}\right)|\mathscr{I}|^{\ell}, \operatorname{sgn}\left(\mathfrak{I}_{2}\right)|\mathfrak{I}|^{\ell}, \ldots, \operatorname{sgn}\left(\mathfrak{I}_{n}\right)|\mathfrak{I}|^{\ell}\right]^{T}$.

\section{Preliminaries and Problem Formulation}

2.1. Graph Theory. The considered NHRS consists of $N$ robots. An undirected graph $\mathscr{g}=\{v, \mathscr{E}, \mathscr{W}\}$ is employed to model the interactions among the robots, where $v=\{1,2, \ldots, N\}$ denotes the robot set, $\mathscr{E} \subseteq v \times v$ symbolizes the edge set, and $\mathscr{W}=\left[w_{i j}\right]_{N \times N}$ is the nonnegatively weighted adjacency matrix. An undirected edge $\left(v_{j}, v_{i}\right) \in \mathscr{E}$ implies that robots $v_{i}$ and $v_{j}$ can directly receive the information from each other. The neighbour set of the robot $i$ is denoted as $\mathcal{N}_{i}=\{j \in \nu \mid(j, i) \in \mathscr{E}\}$. Generally, when $i \neq j$, one has $w_{i j}>0$ if $j \in \mathcal{N}_{i}$; otherwise, $w_{i j}=0$. Besides, it is assumed that $g$ is a simple graph, namely, $w_{i i}=0$ for all $i \in \nu$. The Laplacian matrix $\mathscr{L}=\left[l_{i j}\right]_{N \times N}$ of $\mathscr{g}$ is defined as $l_{i j}=-w_{i j}, \forall i \neq j$, and $l_{i i}=\sum_{j \in \mathcal{N}_{i}} w_{i j}$, for $i, j \in v$. Taking the virtual leader into consideration, a pinning matrix $\mathscr{B}=\operatorname{diag}\left(b_{1}, b_{2}, \ldots, b_{N}\right)$ is introduced to indicate the interactions between the robots and the leader. Specifically, $b_{i}>0$ if the information of the leader is accessible for the robot $i$; otherwise, $b_{i}=0$. It is noteworthy that the leader will not receive any information from the robots. Then, an usual assumption on the interaction topology is given as follows.

Assumption 1. The communication topology between the robots is undirected. Besides, for each robot, there exists at least one path such that the considered robot can receive the information of the leader, namely, the information of the leader is globally reachable for all robots inside.

2.2. System Formulation. Consider the NHRS with the virtual leader. The dynamics and kinematics of the robot $i$ are given as follows:

$$
\left\{\begin{array}{l}
H_{i}\left(q_{i}\right) \ddot{q}_{i}+C_{i}\left(q_{i}, \dot{q}_{i}\right) \dot{q}_{i}+g_{i}\left(q_{i}\right)=\mu_{i}+d_{i}(t), \\
x_{i}=\psi\left(q_{i}\right), \dot{x}_{i}=J_{i}\left(q_{i}\right) \dot{q}_{i},
\end{array}\right.
$$

where $q_{i} \in \mathbb{R}^{p_{i}}$ denotes the generalized coordinates in the joint space and $\dot{q}_{i}$ and $\ddot{q}_{i}$ are its velocity and acceleration, respectively; $x_{i} \in \mathbb{R}^{d}$ represents the generalized coordinates in the task space with $\dot{x}_{i}$ being its velocity; $H_{i}\left(q_{i}\right) \in \mathbb{R}^{p_{i} \times p_{i}}$, $C_{i}\left(q_{i}, \dot{q}_{i}\right) \in \mathbb{R}^{p_{i} \times p_{i}}$, and $g_{i}\left(q_{i}\right) \in C_{i}\left(q_{i}, \dot{q}_{i}\right) \in \mathbb{R}^{p_{i}}$ symbolize the positive-definite inertia matrix, the Coriolis-centrifugal matrix, and the gravitational torque, respectively; $\mu_{i}$ denotes the input torque; $d_{i}(t)$ is the external disturbance; and $J_{i}\left(q_{i}\right)=\partial \psi\left(q_{i}\right) / \partial q_{i} \in g_{i}\left(q_{i}\right) \in \mathbb{R}^{d \times p_{i}}$ represents the Jacobian matrix, which is supposed to be bounded and nonsingular throughout the full text.

Besides, some properties associated with system (1) are presented as follows for the later analysis.

Property 1. $H_{i}\left(q_{i}\right)$ is positive definite, and $\dot{H}_{i}\left(q_{i}\right)-2 C_{i}\left(q_{i}, \dot{q}_{i}\right)$ is skew symmetric, namely, for any 
given $\xi \in \mathbb{R}^{p_{i}}$, one obtains that $\xi^{T} H_{i}\left(q_{i}\right) \xi>0$ and $\dot{\xi}^{T}\left[\dot{H}_{i}\left(q_{i}\right)-2 C_{i}\left(q_{i}, \dot{q}_{i}\right)\right] \xi=0$.

Property 2. $H_{i}\left(q_{i}\right), C_{i}\left(q_{i}\right)$, and $g_{i}\left(q_{i}\right)$ are bounded for all possible $q_{i}$, i.e., $\underline{\sigma}_{H} I_{p_{i}} \leq H_{i}\left(q_{i}\right) \leq \bar{\sigma}_{H} I_{p_{i}},\left\|C_{i}\left(q_{i}, \dot{q}_{i}\right)\right\| \leq \bar{\sigma}_{C}\left\|\dot{q}_{i}\right\|$, and $\left\|g_{i}\left(q_{i}\right)\right\| \leq \bar{\sigma}_{g}$ for all $i \in \nu$, where $\underline{\sigma}_{H}, \bar{\sigma}_{H}, \bar{\sigma}_{C}$, and $\bar{\sigma}_{g}$ are positive constants.

Property 3. The involved dynamic terms can be linearly parameterized for any given vectors $v$ and $\rho$ with proper dimensions, i.e., $\quad H_{i}\left(q_{i}\right) v+C_{i}\left(q_{i}, \dot{q}_{i}\right) \rho+g_{i}\left(q_{i}\right)=$ $Y_{i}\left(q_{i}, \dot{q}_{i}, \rho, v\right) \vartheta_{i}$, where $Y_{i}\left(q_{i}, \dot{q}_{i}, \rho, v\right)$ is the dynamical regressor and $\vartheta_{i}$ is the unknown constant parameter vector.

Remark 1 . Property 3 is obtained because the inertia matrix $H_{i}\left(q_{i}\right)$ can be linearly represented by a set of properly chosen combinations of dynamic parameters. The specific evolutionary process is omitted here for the space limit. Refer to [38] for more details.

On the contrary, the virtual leader is described as

$$
\left\{\begin{array}{l}
\dot{x}_{r}=v_{r} \\
\dot{v}_{r}=a_{r}
\end{array}\right.
$$

where $x_{r}, v_{r}$, and $a_{r}$, respectively, denote the reference position, velocity, and acceleration of the leader. In practical robotic applications, NHRSs are always expected to track the certain desired trajectory with respect to the practical task. Then, a reasonable assumption on the acceleration of the leader is given as follows.

Assumption 2. It is assumed that the jerk of the virtual leader is bounded, namely, $\left\|\dot{a}_{r}\right\| \leq \bar{\sigma}_{\dot{a}}$, where $\bar{\sigma}_{\dot{a}}$ is a positive constant.

2.3. Problem Formulation. The control objective is to design an appropriate control algorithm aiming at solving the timevarying formation tracking problem defined hereinafter. Firstly, the time-varying formation offset of the $i$-th robot is specified by $h_{i}(t) \in \mathbb{R}^{d}$, and the tracking errors are predefined as $e_{i}(t)=x_{i}(t)-x_{r}(t)-h_{i}(t)$ and $\dot{e}_{i}(t)=\dot{x}_{i}(t)-$ $v_{r}(t)-\dot{h}_{i}(t)$.

Definition 1. The time-varying formation tracking problem of the NHRS is addressed if the tracking errors satisfy

$$
\left\{\begin{array}{l}
\lim _{t \longrightarrow \infty}\left\|e_{i}(t)\right\|=0, \\
\lim _{t \longrightarrow \infty}\left\|\dot{e}_{i}(t)\right\|=0,
\end{array}\right.
$$

where $i \in v$.
Some useful lemmas that will be invoked in the later analysis are presented as follows.

Lemma 1 (see [39]). Given the second-order dynamical system

$$
\begin{aligned}
& \dot{\xi}=\eta, \\
& \dot{\eta}=-\Gamma\left[\alpha \operatorname{sig}(\xi)^{\rho_{1}}+\beta \operatorname{sig}(\eta)^{\rho_{2}}\right],
\end{aligned}
$$

where $\xi, \eta \in \mathbb{R}^{n}$ and $\Gamma$ is a positive-definite matrix with proper dimension. Then, the origin is a finite-time stable equilibrium of system (4) if $\alpha>0, \beta>0,0<\rho_{1}<1$, and $\rho_{2}=2 \rho_{1} /\left(\rho_{1}+1\right)$.

Lemma 2 (see [40]). For given $f(t, x, \mu)$ being Lipschitz, the system $\dot{x}=f(t, x, \mu)$ is input-to-state stable, if the corresponding unexcited system $\dot{x}=f(t, x, 0)$ is globally exponentially stable with the equilibrium being the origin, i.e., $x(t)=0$ as $t \longrightarrow \infty$ if $\mu(t) \longrightarrow 0$ as $t \longrightarrow \infty$.

\section{Analysis for Time-Varying Formation Tracking}

3.1. Estimator-Based Hierarchical Cooperative Algorithm I. In this section, EBHC algorithm I is proposed for addressing the time-varying formation tracking problem of NHRSs. For the sake of simplification, the redundant robot set and nonredundant robot set are severally defined as $\rceil=\left\{i \in \nu \mid p_{i}>d\right\}$ and $\beth=\left\{i \in \nu \mid p_{i}=d\right\}$. Furthermore, $J_{i}^{\#}$ is predefined as

$$
J_{i}^{\#}= \begin{cases}J_{i}^{-1}, & i \in \sqsupset, \\ J_{i}^{\dagger}, & i \in\urcorner .\end{cases}
$$

Let $\widehat{x}_{i}, \widehat{v}_{i}, \widehat{a}_{i}$, and $\widehat{\vartheta}_{i}$, respectively, be the estimators of $x_{i}$, $v_{i}, a_{i}$, and $a_{i}, \forall i \in \nu$, where $v_{i}$ and $a_{i}$ are, respectively, the velocity and acceleration in the task space. An auxiliary joint-space velocity is properly defined as

$$
\dot{\widehat{\psi}}_{i}=J_{i}^{\#}\left(\widehat{v}_{i}-\omega_{i}\left(x_{i}-\widehat{x}_{i}\right)\right)+\left(I_{p_{i}}-J_{i}^{\#} J_{i}\right) \varphi_{i},
$$

where $\omega_{i}$ is a positive scalar and $\varphi_{i} \in \mathbb{R}^{p_{i}}$ denotes the negative gradients of some performance indices with respect to the redundant robots. It follows that the auxiliary acceleration can be presented as

$$
\begin{aligned}
\ddot{\widehat{\psi}}_{i}= & \dot{J}_{i}^{\#}\left(\widehat{v}_{i}-\omega_{i}\left(x_{i}-\widehat{x}_{i}\right)\right)+J_{i}^{\#}\left(\widehat{a}_{i}-\omega_{i}\left(\dot{x}_{i}-\widehat{v}_{i}\right)\right) \\
& +\frac{d}{d t}\left[\left(I_{p_{i}}-J_{i}^{\#} J_{i}\right) \varphi_{i}\right] .
\end{aligned}
$$

Thereupon, the sliding vector is defined as

$$
\widehat{s}_{i}=\dot{q}_{i}-\dot{\widehat{\psi}}_{i} .
$$

Then, EBHC algorithm I is presented as follows: 


$$
\begin{aligned}
& \left\{\begin{array}{l}
\mu_{i}=Y_{i}\left(q_{i}, \dot{q}_{i}, \dot{\widehat{\psi}}_{i}, \ddot{\widehat{\psi}}_{i}\right) \widehat{\vartheta}_{i}-J_{i}^{T} \kappa_{o i} J_{i} \widehat{s}_{i}-\kappa_{s i} \widehat{s}_{i}-\kappa_{d i} \operatorname{sgn}\left(\widehat{s}_{i}\right), \\
\dot{\widehat{\vartheta}}_{i}=-\Lambda_{i} Y_{i}^{T}\left(q_{i}, \dot{q}_{i}, \dot{\widehat{\psi}}_{i}, \ddot{\hat{\psi}}_{i}\right) \widehat{\widehat{s}}_{i},
\end{array}\right. \\
& \left\{\begin{array}{l}
\dot{\hat{x}}_{i}=\widehat{v}_{i}, \\
\dot{\hat{v}}_{i}=-\alpha \operatorname{sig}\left(\sum_{j \in \mathcal{N}_{i}} w_{i j}\left(\widehat{x}_{i}-\widehat{x}_{j}+h_{j}-h_{i}\right)+b_{i}\left(\widehat{x}_{i}-x_{r}-h_{i}\right)\right)^{\rho_{1}} \\
-\beta \operatorname{sig}\left(\sum_{j \in \mathcal{N}_{i}} w_{i j}\left(\widehat{v}_{i}-\widehat{v}_{j}+\dot{h}_{j}-\dot{h}_{i}\right)+b_{i}\left(\widehat{v}_{i}-v_{r}-\dot{h}_{i}\right)\right)^{\rho_{2}}+\widehat{a}_{i}+\ddot{h}_{i}, \\
\dot{\hat{a}}_{i}=-\gamma \operatorname{sgn}\left(\sum_{j \in \mathcal{N}_{i}} w_{i j}\left(\widehat{a}_{i}-\widehat{a}_{j}\right)+b_{i}\left(\widehat{a}_{i}-a_{r}\right)\right),
\end{array}\right.
\end{aligned}
$$

where $i \in \nu, \kappa_{o i} \in \mathbb{R}^{d \times d}$, and $\kappa_{s i}, \kappa_{d i} \in \mathbb{R}^{p_{i} \times p_{i}}$ are the feedback gain matrix, $\Lambda_{i}$ is the adaptive positive-definite matrix with appropriate dimensions, and $\alpha, \beta, \gamma, \rho_{1}$, and $\rho_{2}$ are positive constants. To be specific, (9) is the adaptive smooth control layer and (10) is the distributed estimator layer.
Let $\tilde{x}_{i}=\widehat{x}_{i}-x_{r}-h_{i}, \tilde{v}_{i}=\widehat{v}_{i}-v_{r}-\dot{h}_{i}, \tilde{a}_{i}=\widehat{a}_{i}-a_{r}$, and $\widetilde{\vartheta}_{i}=\vartheta_{i}-\widehat{\vartheta}_{i}$. Substituting (9) and (10) into (1) yields the following cascade closed-loop system:

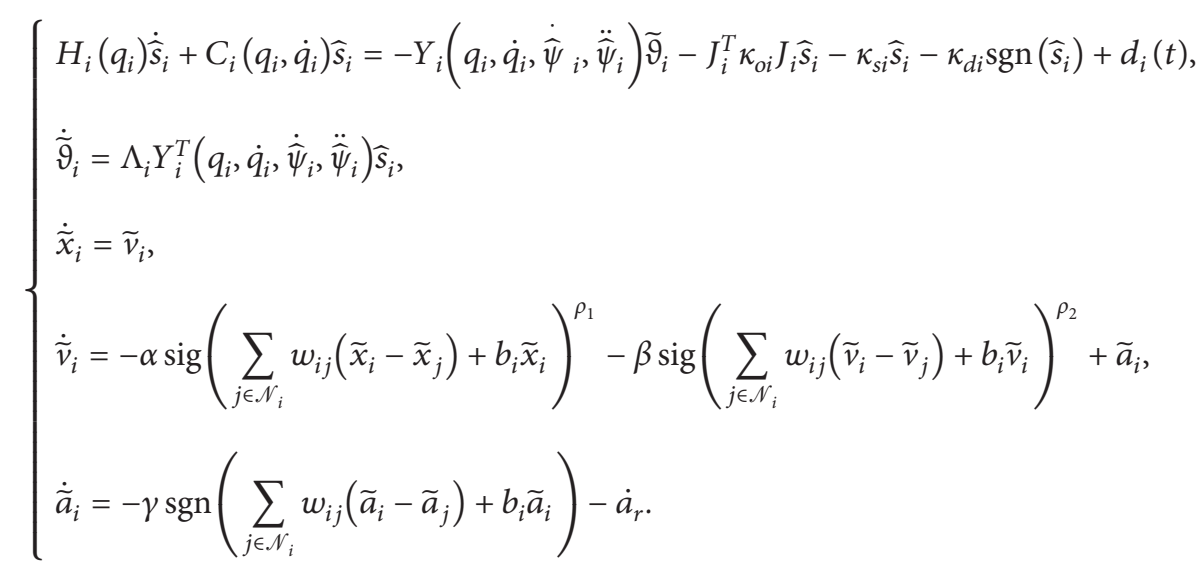

3.2. Coordination Analysis and Boundedness Analysis for the Estimator Layer. Let $\widetilde{x}=\operatorname{col}\left(\widetilde{x}_{1}, \widetilde{x}_{2}, \ldots, \widetilde{x}_{N}\right), \widetilde{v}=\operatorname{col}\left(\widetilde{v}_{1}\right.$, $\left.\tilde{v}_{2}, \ldots, \widetilde{v}_{N}\right), \quad \tilde{a}=\operatorname{col}\left(\tilde{a}_{1}, \tilde{a}_{2}, \ldots, \tilde{a}_{N}\right), a_{R}=1_{N} \otimes a_{r}$, and $\mathscr{J}=\mathscr{L}+\mathscr{B}$. Then, the closed-loop system of the estimator layer can be further rewritten in the following compact form:

$$
\left\{\begin{array}{l}
\dot{\tilde{x}}=\widetilde{v} \\
\dot{\tilde{v}}=-\alpha \operatorname{sig}\left(\left(\mathscr{J} \otimes I_{d}\right) \tilde{x}\right)^{\rho_{1}}-\beta \operatorname{sig}\left(\left(\mathscr{J} \otimes I_{d}\right) \widetilde{v}\right)^{\rho_{2}}+\tilde{a}, \\
\dot{\tilde{a}}=-\gamma \operatorname{sgn}\left(\left(\mathscr{J} \otimes I_{d}\right) \tilde{a}\right)-\dot{a}_{R} .
\end{array}\right.
$$

Theorem 1. Suppose that Assumptions 1 and 2 hold. The distributed estimator algorithm (10) guarantees that there exists $T_{f}>0$ such that $\widetilde{x}_{i}=0$ and $\widetilde{v}_{i}=0$ when $t \geq T_{f}$ for all $i \in \nu$ if

$$
\begin{aligned}
& \alpha>0, \\
& \beta>0, \\
& \gamma>\bar{\sigma}_{\dot{a}}, \\
& 0<\rho_{1}<1, \\
& \rho_{2}=\frac{2 \rho_{1}}{\rho_{1}+1} .
\end{aligned}
$$

Besides, the states $q_{i}$ and $\dot{q}_{i}$ remain bounded when $t_{0} \leq t<T_{f}$ for any given bounded initial values, where $t_{0}$ denotes the initial time.

Proof. For the first presentation, we are going to prove that $\tilde{x}_{i}$ and $\widetilde{v}_{i}, \forall i \in v$, converge to the origin in finite time $T_{f} \geq t_{0}$. 
Based on Assumption 1, it can be derived that $\mathscr{F}$ is positive definite. Then, the Lyapunov function is constructed for the third differential system in (12) as $V_{1}(\widetilde{a})=(1 / 2) \widetilde{a}^{T}\left(\mathscr{J} \otimes I_{d}\right) \widetilde{a}$. It follows that

$$
\begin{aligned}
\dot{V}_{1}(\widetilde{a}) & =\tilde{a}^{T}\left(\mathscr{J} \otimes I_{d}\right) \dot{\tilde{a}} \\
& =-\gamma\left\|\left(\mathscr{J} \otimes I_{d}\right) \tilde{a}\right\|_{1}-\tilde{a}^{T}\left(\mathscr{J} \otimes I_{d}\right) \dot{a}_{R} \\
& \leq-\gamma\left\|\left(\mathscr{g} \otimes I_{d}\right) \tilde{a}\right\|+\left\|\dot{a}_{R}\right\|\left\|\left(\mathscr{J} \otimes I_{d}\right) \tilde{a}\right\| \\
& \leq-\left(\gamma-\bar{\sigma}_{\dot{a}}\right)\left\|\left(\mathscr{J} \otimes I_{d}\right) \tilde{a}\right\| .
\end{aligned}
$$

Equation (13) implies that $\dot{V}_{1}(\widetilde{a}) \leq 0$. Besides, note that $V_{1}(\tilde{a}) \leq(1 / 2)\left\|\mathscr{J} \otimes I_{d}\right\|\|\tilde{a}\|^{2}$. It then follows that $\dot{V}_{1}(\widetilde{a}) \leq-\left(\gamma-\bar{\sigma}_{\dot{a}}\right) \sqrt{2\left\|\mathscr{J} \otimes I_{d}\right\|} V_{1}^{1 / 2}(\widetilde{a})$. Thus, we can derive that $\widetilde{a}$ converges to zero in finite time $\mathscr{J}_{f 1}$. Then, when $t \geq \mathscr{J}_{f 1}$, (12) equals

$$
\left\{\begin{array}{l}
\dot{\tilde{x}}=\widetilde{v} \\
\dot{\tilde{v}}=-\alpha \operatorname{sig}\left(\left(\mathscr{g} \otimes I_{d}\right) \widetilde{x}\right)^{\rho_{1}}-\beta \operatorname{sig}\left(\left(\mathscr{g} \otimes I_{d}\right) \widetilde{v}\right)^{\rho_{2}} .
\end{array}\right.
$$

Taking the linear transformation for $\widetilde{X}=\left(\mathscr{J} \otimes I_{d}\right) \widetilde{x}$ and $\widetilde{V}=\left(\mathscr{g} \otimes I_{d}\right) \widetilde{v}$ yields that

$$
\left\{\begin{array}{l}
\dot{\tilde{X}}=\tilde{V} \\
\dot{\widetilde{V}}=-\left(\mathscr{J} \otimes I_{d}\right)\left[\alpha \operatorname{sig}(\widetilde{X})^{\rho_{1}}+\beta \operatorname{sig}(\widetilde{V})^{\rho_{2}}\right] .
\end{array}\right.
$$

Then, according to Lemma 1 and (13), system (16) is globally finite-time stable with the origin being its stable equilibrium, i.e., there exists a settling time $T_{f 2}>0$ such that $\widetilde{X}$ and $\widetilde{V}$ converge to the origin on $t \in\left[\mathscr{F}_{f 1}, \mathscr{F}_{f 2}\right]$. Note that $\mathscr{J}_{f}=\mathscr{J}_{f 1}+\mathscr{J}_{f 2}$. It follows that $\tilde{x}=0$ and $\widetilde{v}=0$ when $t \geq T_{f}$, namely, $\tilde{x}_{i}=0$ and $\widetilde{v}_{i}=0$ when $t \geq T_{f}$ for all $i \in \nu$.

The second presentation focuses on the boundedness analysis. Note that

$$
\begin{gathered}
\left\|\operatorname{sgn}\left(\sum_{j \in \mathcal{N}_{i}} w_{i j}\left(\hat{x}_{i}-\hat{x}_{j}+h_{j}-h_{i}\right)+b_{i}\left(\hat{x}_{i}-x_{r}-h_{i}\right)\right)\right\|_{\infty} \leq 1, \\
\left\|\operatorname{sgn}\left(\sum_{j \in \mathcal{N}_{i}} w_{i j}\left(\hat{v}_{i}-\widehat{v}_{j}+\dot{h}_{j}-\dot{h}_{i}\right)+b_{i}\left(\widehat{v}_{i}-v_{r}-\dot{h}_{i}\right)\right)\right\|_{\infty} \leq 1 .
\end{gathered}
$$

Besides, Assumption 2 implies that $x_{r}, v_{r}$, and $a_{r}$ are all globally bounded on $t \in\left[t_{0}, T_{f}\right]$, namely, $x_{r}, v_{r}$, $a_{r} \in \mathscr{L}_{2} \cap \mathscr{L}_{\infty}$ as $t_{0} \leq t<T_{f}$, and the formation offsets of robots are usually set to be bounded in practical robotic applications, i.e., $h_{i}(t), \dot{h}_{i}(t), \ddot{h}_{i}(t) \in \mathscr{L}_{2} \cap \mathscr{L}_{\infty}$ for all $i \in v$. For any given initial values $\hat{x}_{i}\left(t_{0}\right), \widehat{v}_{i}\left(t_{0}\right) \in \mathscr{L}_{2} \cap \mathscr{L}_{\infty},(10)$ implies that $\hat{x}_{i}, \widehat{v}_{i} \in \mathscr{L}_{2} \cap \mathscr{L}_{\infty}$ when $t_{0} \leq t<T_{f}$. Besides, through (1), we know that $x_{i}, \dot{x}_{i} \in \mathscr{L}_{2} \cap \mathscr{L}_{\infty}$ since $q_{i}, \dot{q}_{i} \in \mathscr{L}_{2} \cap \mathscr{L}_{\infty}$ as $t_{0} \leq t<T_{f}$. Then, substituting the aforementioned variables into (6)-(8) yields that $\widehat{\psi}_{i}, \dot{\hat{\psi}}_{i}, \widehat{s}_{i} \in \mathscr{L}_{2} \cap \mathscr{L}_{\infty}$ as $t_{0} \leq t<T_{f}$. It follows from Property 2 that $Y_{i}\left(q_{i}, \dot{q}_{i}, \widehat{\psi}_{i}, \widehat{\psi}_{i}\right) \in \mathscr{L}_{2} \cap \mathscr{L}_{\infty}$ as $t_{0} \leq t<T_{f}$. Furthermore, (9) implies that $\widehat{\vartheta}_{i} \in \mathscr{L}_{2} \cap \mathscr{L}_{\infty}$ as $t_{0} \leq t<T_{f}$ for any given initial value $\widehat{\vartheta}_{i}\left(t_{0}\right) \in \mathscr{L}_{2} \cap \mathscr{L}_{\infty}$. Hence, we can conclude that $q_{i}, \dot{q}_{i}, \hat{s}_{i}, \widehat{\vartheta}_{i} \in \mathscr{L}_{2} \cap \mathscr{L}_{\infty}$ if the initial values $q_{i}\left(t_{0}\right), \dot{q}_{i}\left(t_{0}\right), \hat{x}_{i}\left(t_{0}\right), \hat{x}\left(t_{0}\right), \widehat{\vartheta}_{i}\left(t_{0}\right) \in \mathscr{L}_{2} \cap \mathscr{L}_{\infty} \quad$ as $\quad t_{0} \leq t<$ $T_{f}, \forall i \in \nu$. This completes the proof.

3.3. Convergence Analysis. In this section, we will show whether the time-varying formation tracking problem can be addressed under the proposed EBHC algorithm I or not. Firstly, the norm variables associated with $\widehat{\psi}_{i}, \ddot{\hat{\psi}}_{i}$, and $\widehat{s}_{i}$ are given as

$$
\begin{aligned}
\dot{\psi}_{i}= & J_{i}^{\#}\left(v_{r}+\dot{h}_{i}-\omega_{i}\left(x_{i}-x_{r}-h_{i}\right)\right)+\left(I_{p_{i}}-J_{i}^{\#} J_{i}\right) \varphi_{i}, \\
\ddot{\psi}_{i}= & \dot{J}_{i}^{\#}\left(v_{r}+\dot{h}_{i}-\omega_{i}\left(x_{i}-x_{r}-h_{i}\right)\right)+J_{i}^{\#}\left(a_{r}-\omega_{i}\right. \\
& \left.\cdot\left(\dot{x}_{i}-v_{r}-\dot{h_{i}}\right)\right)+\frac{\mathrm{d}}{\mathrm{d} t}\left[\left(I_{p_{i}}-J_{i}^{\#} J_{i}\right) \varphi_{i}\right], \\
s_{i}= & q_{i}-\dot{\psi}_{i} .
\end{aligned}
$$

Based on Theorem 1, it can be figured out that $\dot{\psi}_{i}, \ddot{\psi}_{i}$, and $s_{i}$ remain bounded when $t_{0} \leq t<T_{f}$ and $\widehat{\psi}_{i}=\dot{\psi}_{i}, \ddot{\widehat{\psi}}_{i}=\ddot{\psi}_{i}$, and $\widehat{s}_{i}=s_{i}$ when $t \geq T_{f}$. Thus, when $t \geq T_{f}$, the following closedloop system arises based on (11):

$$
\left\{\begin{array}{l}
\dot{s}_{i}=-H_{i}^{-1}\left(q_{i}\right)\left[C_{i}\left(q_{i}, \dot{q}_{i}\right) s_{i}+Y_{i}\left(q_{i}, \dot{q}_{i}, \dot{\psi}_{i}, \ddot{\psi}_{i}\right) \widetilde{\vartheta}+J_{i}^{T} \kappa_{o i} J_{i} s_{i}\right. \\
\left.+\kappa_{s i} s_{i}+\kappa_{d i} \operatorname{sgn}\left(s_{i}\right)-d_{i}(t)\right], \\
\dot{\widetilde{\vartheta}}_{i}=\Lambda_{i} Y_{i}^{T}\left(q_{i}, \dot{q}_{i}, \dot{\psi}_{i}, \ddot{\psi}_{i}\right) s_{i} .
\end{array}\right.
$$

Theorem 2. Suppose that Assumptions 1 and 2 hold. The time-varying formation tracking problem of the NHRS can be addressed under the proposed EBHC algorithm I, if

$$
\begin{aligned}
& \lambda_{\min }\left(\kappa_{o i}\right)>0, \\
& \lambda_{\min }\left(\kappa_{s i}\right)>0, \\
& \lambda_{\min }\left(\kappa_{d i}\right) \geq \sup _{t \geq t_{0}}\left\|d_{i}(t)\right\|,
\end{aligned}
$$

namely, it can be obtained that the task-space coordinates $x_{i}$ and $v_{i}$ can eventually form the time-varying formations, i.e., $x_{i} \longrightarrow x_{r}+h_{i}(t)$ and $v_{i} \longrightarrow v_{r}+\dot{h}_{i}(t)$ as $t \longrightarrow \infty$.

Proof. Consider the following storage function as the Lyapunov function candidate for system (21):

$$
V\left(s_{i}\right)=\frac{1}{2} s_{i}^{T} H_{i}\left(q_{i}\right) s_{i}+\frac{1}{2} \widetilde{\vartheta}_{i}^{T} \Lambda_{i}^{-1} \widetilde{\vartheta}_{i} .
$$


Differentiating (23) along (21) yields that

$$
\begin{aligned}
\dot{V}\left(s_{i}\right)= & s_{i}^{T} H_{i}\left(q_{i}\right) \dot{s}_{i}+\frac{1}{2} s_{i}^{T} \dot{H}_{i}\left(q_{i}\right) s_{i}+\widetilde{\vartheta}_{i}^{T} \Lambda_{i}^{-1} \dot{\widetilde{\vartheta}}_{i} \\
= & -s_{i}^{T}\left[C_{i}\left(q_{i}, \dot{q}_{i}\right) s_{i}+Y_{i}\left(q_{i}, \dot{q}_{i}, \dot{\psi}_{i}, \ddot{\psi}_{i}\right) \widetilde{\vartheta}+J_{i}^{T} \kappa_{o i} J_{i} s_{i}\right. \\
& \left.+\kappa_{s i} s_{i}+\kappa_{d i} \operatorname{sgn}\left(s_{i}\right)-d_{i}(t)\right]+\frac{1}{2} s_{i}^{T} \dot{H}_{i}\left(q_{i}\right) s_{i}+\widetilde{\vartheta}_{i}^{T} Y_{i}^{T} \\
& \cdot\left(q_{i}, \dot{q}_{i}, \dot{\psi}_{i}, \ddot{\psi}_{i}\right) s_{i} \\
= & \frac{1}{2} s_{i}^{T}\left[\dot{H}_{i}\left(q_{i}\right)-2 C_{i}\left(q_{i}, \dot{q}_{i}\right)\right] s_{i} \\
& -s_{i}^{T}\left(J_{i}^{T} \kappa_{o i} J_{i}+\kappa_{s i}\right) s_{i}-s_{i}^{T} \kappa_{d i} \operatorname{sgn}\left(s_{i}\right)+s_{i}^{T} d_{i}(t) \\
\leq & -s_{i}^{T}\left(J_{i}^{T} \kappa_{o i} J_{i}+\kappa_{s i}\right) s_{i}-\left\|s_{i}\right\|\left[\lambda_{\min }\left(\kappa_{d i}\right)-\sup _{t \geq t_{0}}\left\|d_{i}(t)\right\|\right]
\end{aligned}
$$

where Property 1 and $\lambda_{\min }\left(\kappa_{d i}\right) \geq \sup _{t \geq t_{0}}\left\|d_{i}(t)\right\|$ in (22) have been invoked to make the above inequality hold. Thereupon, $\lambda_{\text {min }}\left(\kappa_{o i}\right)>0$ and $\lambda_{\min }\left(\kappa_{s i}\right)>0$ imply that $\dot{V}\left(s_{i}\right)<0$. Furthermore, (22) and (24) provide that $s_{i} \in \mathscr{L}_{2} \cap \mathscr{L}_{\infty}$. Then, one obtains that $\dot{s}_{i} \in \mathscr{L}_{\infty}$ through the closed-loop system (21). Thus, $\ddot{V}\left(s_{i}\right) \in \mathscr{L}_{\infty}$ is derived. According to Barbalat's lemma [41] and the above stability analysis, it thus can be concluded that $s_{i} \longrightarrow 0$ as $t \longrightarrow \infty$.

According to (18) and (20), $J_{i}\left(q_{i}\right) s_{i}$ is presented as follows when $t \geq T_{f}$ :

$$
\begin{aligned}
J_{i}\left(q_{i}\right) s_{i} & =J_{i}\left(q_{i}\right) q_{i}-\left[v_{r}+\dot{h}_{i}-\omega_{i}\left(x_{i}-x_{r}-h_{i}\right)\right] \\
& =\dot{x}_{i}-v_{r}-\dot{h}_{i}+\omega_{i}\left(x_{i}-x_{r}-h_{i}\right) \\
& =\dot{e}_{i}+\omega_{i} e_{i},
\end{aligned}
$$

which can be further rewritten in the following form:

$$
\dot{e}_{i}+\omega_{i} e_{i}=J_{i}\left(q_{i}\right) s_{i} \text {. }
$$

Note that $J_{i}\left(q_{i}\right) s_{i} \longrightarrow 0$ as $t \longrightarrow \infty$ for the boundedness of $J_{i}\left(q_{i}\right)$. According to Lemma 2, it thus follows that the kinematics loop (26) is input-to-state stable with $J_{i}\left(q_{i}\right) s_{i}$ being the input and $e_{i}$ and $\dot{e}_{i}$ being the states. Then, $\forall i \in v$, we draw the conclusion that $e_{i} \longrightarrow 0$ and $\dot{e}_{i} \longrightarrow 0$ as $t \longrightarrow \infty$ through the fact that $J_{i}\left(q_{i}\right) s_{i} \longrightarrow 0$ as $t \longrightarrow \infty$. This completes the proof.

Remark 2. Selecting appropriate $\varphi_{i}$ for a redundant robot can execute some subtasks but does not conflict with the main task (i.e., the time-varying formation tracking task). For instants, denote $q_{i}(1)$ as the first element of $q_{i}, i \in \neg$, and choose the performance index as $H_{i}\left(q_{i}\right)=0.4\left(12-q_{i}(1)\right)^{2}$, then one has $\varphi_{i}=-\left(\partial H_{i}\left(q_{i}\right) / \partial q_{i}\right)=[0.8(12-$ $\left.\left.q_{i}(1)\right), 0, \ldots, 0\right]^{T}$. Such a setting mainly aims at forcing the first joint of the $i$-th robot toward $12 \mathrm{rad}$. A corresponding simulation experiment on redundant robots will be conducted to support the above presentation.
Remark 3. According to the study in [32], the subtask for the redundant robots is called completed if

$$
\lim _{t \longrightarrow \infty} e_{s i}=\lim _{t \longrightarrow \infty}\left[I_{p_{i}}-J_{i}^{\#}\left(q_{i}\right) J_{i}\left(q_{i}\right)\right]\left(\dot{q}_{i}-\varphi_{i}\right)=0 .
$$

Then, it will be discussed whether the subtasks can be addressed or not under the proposed EBHC algorithm I. Note that when $t \geq T_{f}$,

$$
\begin{aligned}
e_{s i}= & {\left[I_{p_{i}}-J_{i}^{\#}\left(q_{i}\right) J_{i}\left(q_{i}\right)\right]\left(\dot{q}_{i}-\varphi_{i}\right) } \\
= & {\left[I_{p_{i}}-J_{i}^{\#}\left(q_{i}\right) J_{i}\left(q_{i}\right)\right]\left[\dot{q}_{i}-J_{i}^{\#}\left(v_{r}+\dot{h}_{i}-\omega_{i}\left(x_{i}-x_{r}-h_{i}\right)\right)\right.} \\
& \left.-\left(I_{p_{i}}-J_{i}^{\#}\left(q_{i}\right)\right) \varphi_{i}\right] \\
= & {\left[I_{p_{i}}-J_{i}^{\#}\left(q_{i}\right) J_{i}\left(q_{i}\right)\right]\left(\dot{q}_{i}-\dot{\psi}_{i}\right) } \\
= & {\left[I_{p_{i}}-J_{i}^{\#}\left(q_{i}\right) J_{i}\left(q_{i}\right)\right] s_{i}, }
\end{aligned}
$$

where $\quad\left[I_{p_{i}}-J_{i}^{\#} \quad\left(q_{i}\right) J_{i}\left(q_{i}\right)\right]\left[I_{p_{i}}-J_{i}^{\#}\left(q_{i}\right) J_{i}\left(q_{i}\right)\right]=\left[I_{p_{i}}-\right.$ $\left.J_{i}^{\#}\left(q_{i}\right) J_{i}\left(q_{i}\right)\right], \quad\left[I_{p_{i}}-J_{i}^{\#}\left(q_{i}\right) J_{i}\left(q_{i}\right)\right] J_{i}^{\#}\left(q_{i}\right)=0$, and (18) and (20) have been invoked to obtain the above equation. It has been already obtained from Theorem 2 that $s_{i} \longrightarrow 0$ as $t \longrightarrow \infty$. Then, one can easily derive that $\left[I_{p_{i}}-J_{i}^{\#}\left(q_{i}\right) J_{i}\left(q_{i}\right)\right] s_{i} \longrightarrow 0 \quad$ as $t \longrightarrow \infty$, namely, $\lim _{t \rightarrow \infty} e_{s i}=0$. This completes the proof.

Remark 4. Referencing [32], we can choose the performance index of redundant robots as $H_{i}\left(q_{i}\right)=-\operatorname{det}\left(J_{i} J_{i}^{T}\right)$ to maximize the manipulability of the redundant robots and tend to keep the robots away from singularities, namely, setting $\varphi_{i}=$ - $\left(\partial H_{i}\left(q_{i}\right) / \partial q_{i}\right)=\left(\partial \operatorname{det}\left(J_{i} J_{i}^{T}\right) / \partial q_{i}\right)$ can help maintain the Jacobian matrices for the redundant robots to be nonsingular.

\section{Further Results on Multiple Time-Varying Formation Tracking}

In this section, a similar controller will be designed to address the multiple time-varying formation tracking problem of NHRSs.

Firstly, some different definitions on graph theory should be further presented here. The adjacency matrix $\mathscr{W}$ is no longer nonnegatively weighted here. If the robot $i$ cooperates with the robot $j$, then $w_{i j}=w_{j i}>0$; if the robot $i$ competes with the robot $j$, then $w_{i j}=w_{j i}<0$. Besides, the considered NHRS can be divided into $M$ subnetworks. The undirected graph $\mathscr{L}_{k}=\left\{v_{k}, \mathscr{E}_{k}, \mathscr{W}_{k}\right\}, \quad k \in\{1,2, \ldots, M\}$, which is nonnegatively weighted, is employed to describe the interaction of the $k$-th subnetwork. Besides, each robot can only belong to one of these subnetworks, namely, $v_{k} \cap \nu_{p}=$ $\varnothing$ for $k \neq p \in\{1,2, \ldots, M\}$ and $\cup_{i=1}^{M} \nu_{i}=v . n_{k}$ is utilized to denote the number of robots in the $k$-th subnetwork. Each subnetwork corresponds to a single virtual leader. Taking the $M$ virtual leaders into consideration, the pinning matrix $\mathscr{B}_{k}$ is employed to illustrate the interactions between the robots in the subnetwork $k$ and their corresponding leader. Then, 
$\mathscr{B}=\operatorname{diag}\left(\mathscr{B}_{1}, \mathscr{B}_{2}, \ldots, \mathscr{B}_{M}\right)$. Thereupon, two different assumptions on the interaction topology are given as follows.

Assumption 3. For each subnetwork with the corresponding leader, there exists at least one path, through which the information of the leader can be obtained by the robots inside.

Assumption 4. The total information that each robot receives from any other different subnetworks is zero, i.e., $\sum_{j=\underline{r_{k}}}^{\overline{r_{k}}} l_{i j}=0, \quad \forall i \in v_{p}, \quad k \in\{1,2, \ldots, M\}-\{p\}, \quad$ where $\underline{r_{k}}=1+\sum_{i=1}^{k} n_{i-1}$ and $\overline{r_{k}}=\sum_{i=1}^{k} n_{i}$. Note that $n_{0}=0$.

The virtual leader associated with the $k$-th subnetwork is described as

$$
\left\{\begin{array}{l}
\dot{x}_{r, k}=v_{r, k} \\
\dot{v}_{r, k}=a_{r, k}
\end{array}\right.
$$

where $x_{r, k}, v_{r, k}$, and $a_{r, k}$, respectively, denote the position, velocity, and acceleration of the $k$-th leader, $k \in\{1,2, \ldots, M\}$.

Assumption 5. The jerks of all the leaders are bounded, namely, there exists a uniform upper bound $\bar{\sigma}_{\dot{a}_{r}}$ such that $\left\|a_{r, k}\right\| \leq \bar{\sigma}_{a_{r}}, \forall k \in\{1,2, \ldots, M\}$.
The control objective in this section mainly focuses on the multiple time-varying formation tracking problem of the NHRS defined hereinafter.

Definition 2. The multiple time-varying formation tracking problem of the NHRS is addressed if the tracking errors satisfy

$$
\left\{\begin{array}{l}
\lim _{t \longrightarrow \infty}\left\|e_{i, k}(t)\right\|=0, \\
\lim _{t \longrightarrow \infty}\left\|\dot{e}_{i, k}(t)\right\|=0,
\end{array}\right.
$$

for all $i \in v_{k}, k \in\{1,2, \ldots, M\}$, where $e_{i, k}(t)=x_{i}-x_{r, k}-h_{i}$ and $\dot{e}_{i, k}(t)=\dot{x}_{i}-v_{r, k}-\dot{h}_{i}$ denote the tracking errors.

4.1. Estimator-Based Hierarchical Cooperative Algorithm II. In this section, a similar controller is designed to address the multiple time-varying tracking problem of NHRSs. The definitions of $\neg, \beth, J_{i}^{\#}, \widehat{\psi}_{i}, \ddot{\hat{\psi}}_{i}, \widehat{s}_{i}, \dot{\psi}_{i}, \ddot{\psi}_{i}$, and $s_{i}$ are the same as in Section 3. Then, EBHC algorithm II is presented as follows:

$$
\begin{aligned}
& \left\{\begin{array}{l}
\mu_{i}=Y_{i}\left(q_{i}, \dot{q}_{i}, \dot{\widehat{\psi}}_{i}, \ddot{\widehat{\psi}}_{i}\right) \widehat{\vartheta}_{i}-J_{i}^{T} \kappa_{o i} J_{i} \widehat{s}_{i}-\kappa_{s i} \widehat{s}_{i}-\kappa_{d i} \operatorname{sgn}\left(\widehat{s}_{i}\right), \\
\dot{\widehat{\vartheta}}_{i}=-\Lambda_{i} Y_{i}^{T}\left(q_{i}, \dot{q}_{i}, \dot{\hat{\psi}}_{i}, \ddot{\widehat{\psi}}_{i}\right) \widehat{\widehat{s}}_{i},
\end{array}\right. \\
& \left\{\begin{array}{l}
\dot{\hat{x}}_{i}=\widehat{v}_{i}, \\
\dot{\hat{v}}_{i}=-\alpha \operatorname{sig}\left(\sum_{j \in \mathcal{N}_{i}} w_{i j}\left(\widehat{x}_{i}-\widehat{x}_{j}+h_{j}-h_{i}\right)+b_{i}\left(\widehat{x}_{i}-x_{r, k}-h_{i}\right)\right)^{\rho_{1}} \\
-\beta \operatorname{sig}\left(\sum_{j \in \mathcal{N}_{i}} w_{i j}\left(\widehat{v}_{i}-\widehat{v}_{j}+\dot{h}_{j}-\dot{h}_{i}\right)+b_{i}\left(\widehat{v}_{i}-v_{r, k}-\dot{h}_{i}\right)\right)^{\rho_{2}}+\widehat{a}_{i}+\ddot{h}_{i} \\
\dot{\hat{a}}_{i}=-\gamma \operatorname{sgn}\left(\sum_{j \in \mathcal{N}_{i}} w_{i j}\left(\widehat{a}_{i}-\widehat{a}_{j}\right)+b_{i}\left(\widehat{a}_{i}-a_{r, k}\right)\right),
\end{array}\right.
\end{aligned}
$$

where $i \in v_{k}, k \in\{1,2, \ldots, M\}$, and the remaining control parameters are the same as in (9) and (10).

4.2. Convergence Analysis for Multiple Time-Varying Tracking. In this section, it will be tested whether the multiple time-varying tracking problem of NHRSs can be solved or not. Redefine $\tilde{x}_{i, k}=\widehat{x}_{i}-x_{r, k}-h_{i}$, $\widetilde{v}_{i, k}=\widehat{v}_{i}-v_{r, k}-\dot{h}_{i}$, and $\tilde{a}_{i, k}=\widehat{a}_{i}-a_{r, k}, i \in v_{k}$. Based on Assumption 4, substituting (31) and (32) into (1) yields the following cascade closed-loop system: 


$$
\left\{\begin{array}{l}
H_{i}\left(q_{i}\right) \dot{\widehat{s}}_{i}+C_{i}\left(q_{i}, \dot{q}_{i}\right) \widehat{s}_{i}=-Y_{i}\left(q_{i}, \dot{q}_{i}, \dot{\widehat{\psi}}_{i}, \ddot{\widehat{\psi}}_{i}\right) \widetilde{\vartheta}_{i}-J_{i}^{T} \kappa_{o i} J_{i} \widehat{s}_{i} \\
-\kappa_{s i} \widehat{s}_{i}-\kappa_{d i} \operatorname{sgn}\left(\widehat{s}_{i}\right)+\mu_{i, d}, \\
\dot{\widetilde{\vartheta}}_{i}=\Lambda_{i} Y_{i}^{T}\left(q_{i}, \dot{q}_{i}, \dot{\widehat{\psi}}_{i}, \ddot{\widehat{\psi}}_{i}\right) \widehat{s}_{i}, \\
\dot{\tilde{x}}_{i, k}=\widetilde{v}_{i, k}, \\
\dot{\widetilde{v}}_{i, k}=-\alpha \operatorname{sig}\left(\sum_{j \in \mathcal{N}_{i}} w_{i j}\left(\tilde{x}_{i, k}-\tilde{x}_{j, p}\right)+b_{i} \tilde{x}_{i, k}\right)^{\rho_{1}}, \\
-\beta \operatorname{sig}\left(\sum_{j \in \mathcal{N}_{i}} w_{i j}\left(\widetilde{v}_{i, k}-\widetilde{v}_{j, p}\right)+b_{i} \widetilde{v}_{i, k}\right)^{\rho_{2}}+\tilde{a}_{i, k}, \\
\dot{\tilde{a}}_{i, k}=-\gamma \operatorname{sgn}\left(\sum_{j \in \mathcal{N}_{i}} w_{i j}\left(\tilde{a}_{i, k}-\tilde{a}_{j, p}\right)+b_{i} \tilde{a}_{i, k}\right)-\dot{a}_{r},
\end{array}\right.
$$

where $i \in \nu_{k}$ and $j \in \nu_{p}, k, p \in\{1,2, \ldots, M\}$.

Remark 5. Assumption 4 plays an important role in deriving the closed-loop system (33). To be specific, it can be obtained by mathematics deformation that

$$
\begin{aligned}
\sum_{j \in \mathcal{N}_{i}} w_{i j}\left(\hat{x}_{i}-\widehat{x}_{j}+h_{j}-h_{i}\right) & =\sum_{j \in \mathcal{N}_{i}} w_{i j}\left[\left(\hat{x}_{i}-x_{r, k}-h_{i}-\widehat{x}_{j}+x_{r, p}+h_{j}\right)+\left(x_{r, k}-x_{r, p}\right)\right] \\
& =\sum_{j \in \mathcal{N}_{i}} w_{i j}\left(\tilde{x}_{i, k}-\tilde{x}_{j, k}\right)+\left(\mathscr{L}(i,:) \otimes I_{d}\right) x_{R}
\end{aligned}
$$

where $i \in v_{k}$ and $j \in \nu_{p}, k, p \in\{1,2, \ldots, M\}, \mathscr{L}(i,:)$ denotes the $i$-th row vector of $\mathscr{L}$, and

$$
x_{R}=\operatorname{col}(\underbrace{x_{r, 1}, x_{r, 1}, \ldots, x_{r, 1}}_{n_{1}}, \underbrace{x_{r, 2}, x_{r, 2}, \ldots x_{r, 2}}_{n_{2}}, \ldots, \underbrace{x_{r, M}, x_{r, M}, \ldots, x_{r, M}}_{n_{M}}) .
$$

It is noteworthy that Assumption 4 guarantees that $\left(\mathscr{L}(i,:) \otimes I_{d}\right) x_{R}=0$. Besides, similar unitization has been employed in the deformation of $\sum_{j \in \mathcal{N}_{i}} w_{i j}\left(\widehat{v}_{i}-\widehat{v}_{j}+\dot{h}_{j}-\dot{h}_{i}\right)$ and $\sum_{j \in \mathcal{N}_{i}} w_{i j}\left(\widehat{a}_{i}-\widehat{a}_{j}\right)$.

Theorem 3. Suppose Assumptions 3-5 hold, then the multiple time-varying formation tracking problem can be solved under the designed EBHC algorithm II (31) and (32), if (13) and (22) hold and

$$
\min _{k=1}^{N}\left\{\lambda_{k}(\mathscr{L}+\mathscr{B})>0\right\}
$$

namely, $\quad x_{i} \longrightarrow x_{r, k}+h_{i}(t)$ and $v_{i} \longrightarrow v_{r, k}+\dot{h}_{i}(t)$ as $t \longrightarrow \infty$ can be eventually obtained, $\forall i \in v_{k}, k \in\{1,2, \ldots, M\}$.
Proof. Note the adaptive smooth control layer is the same as in the previous part. Thus, we just need to redo the cooperative analysis and boundedness analysis of the distributed estimator layer. Similarly, redefine the following compact vectors:

$$
\begin{gathered}
\tilde{x}=\operatorname{col}\left(\widetilde{x}_{1,1}, \ldots, \widetilde{x}_{n_{1}, 1}, \ldots, \widetilde{x}_{\left(1+\sum_{i=1}^{M-1} n_{i}\right), M}, \ldots, \widetilde{x}_{N, M}\right), \\
\widetilde{v}=\operatorname{col}\left(\widetilde{v}_{1,1}, \ldots, \widetilde{v}_{n_{1}, 1}, \ldots, \widetilde{v}_{\left(1+\sum_{i=1}^{M-1} n_{i}\right), M}, \ldots, \widetilde{v}_{N, M}\right), \\
\widetilde{a}=\operatorname{col}\left(\widetilde{a}_{1,1}, \ldots, \widetilde{a}_{n_{1}, 1}, \ldots, \widetilde{a}_{\left(1+\sum_{i=1}^{M-1} n_{i}\right), M}, \ldots, \widetilde{a}_{N, M}\right) .
\end{gathered}
$$

It thus follows that 


$$
\left\{\begin{array}{l}
\dot{\tilde{x}}=\widetilde{v} \\
\dot{\tilde{v}}=-\alpha \operatorname{sig}\left[\left(\mathscr{J} \otimes I_{d}\right) \tilde{x}\right]^{\rho_{1}}-\beta \operatorname{sig}\left[\left(\mathscr{J} \otimes I_{d}\right) \tilde{v}\right]^{\rho_{2}}+\tilde{a}, \\
\dot{\tilde{a}}=-\gamma \operatorname{sgn}\left(\left(\mathscr{J} \otimes I_{d}\right) \widetilde{a}\right)-\dot{a}_{R},
\end{array}\right.
$$

where $a_{R}=\ddot{x}_{R}\left(x_{R}\right.$ is defined in Remark 5). The fact that $g$ is undirected guarantees the symmetry of $\mathscr{L}$. It thus follows from Assumption 3 and (36) that $\mathscr{J}=\mathscr{L}+\mathscr{B}$ is positive definite. Then, by similar analysis in Theorem 1, it can be derived that $\widehat{x}_{i}$ and $\widehat{v}_{i}$ converge to $x_{r, k}$ and $v_{r, k}$ in finite time, namely, there exists $T_{f}>t_{0}$ such that $\tilde{x}_{i, k}=0$ and $\widetilde{v}_{i, k}=0$, $\forall i \in v_{k}, k \in\{1,2, \ldots, M\}$. Besides, $q_{i}, \dot{q}_{i}, \widehat{s}_{i}, \widehat{\vartheta}_{i} \in \mathscr{L}_{2} \cap \mathscr{L}_{\infty}$ as $t_{0} \leq t \leq T_{f}$.

The remaining convergence analysis of the adaptive smooth control layer is the same as that in Theorem 2 and is omitted here. Besides, the subtasks for the redundant robots can be fulfilled by the analysis in Remark 3 . This completes the proof.

\section{Simulation}

In this section, two simulation examples are provided to illustrate the effectiveness of the proposed EBHC algorithms I and II. The simulation examples hereinafter are performed in the XY plane, and the considered NHRS consists of 2DOF robots and 3-DOF robots, namely, $d=2$ and $p_{i}=2$ or 3 . Besides, the mechanical structures of both the nonredundant robot and redundant robot are shown in Figure 1.

Example 1. EBHC algorithm I (9) and (10) are used for (1) to address the time-varying formation tracking problem of the NHRS consisting of four robots in the case of one virtual leader. It is set that robot 1 is redundant and the remain ones are nonredundant, whose physical parameters are selected as $m_{1}=\operatorname{col}(3.0,2.0,1.0) \mathrm{kg}, \quad l_{1}=\operatorname{col}(5.0,4.0,3.0) \mathrm{m}, \quad$ and $r_{1}=0.5 l_{1}, \quad$ and $\quad m_{i}=\operatorname{col}(3.8+0.1 i, 3.3+0.1 i) \mathrm{kg}$, $l_{i}=\operatorname{col}(6.6+0.2 i, 5.6+0.2 i) \mathrm{m}$, and $r_{i}=0.5 l_{i}$ for $i \in\{2,3,4\}$. The interactions among the NHRS and the virtual leader are shown in Figure 2(a). To be specific, the node $L$ denotes the leader, node 1 represents the redundant robot, nodes 2-4 are the nonredundant robots, and robots 1 and 2 can directly receive the information from the leader. It thus follows that $\mathscr{B}=\operatorname{diag}(2,0,0,3)$ and

$$
\mathscr{L}=\left(\begin{array}{cccc}
1 & -1 & 0 & 0 \\
-1 & 1 & 0 & 0 \\
0 & 0 & 2 & -2 \\
0 & 0 & -2 & 2
\end{array}\right) .
$$

Let the external disturbances be $d_{i}(t)=-0.1[\cos (t), \sin (t)]^{T}$ for 2-DOF robots and $d_{i}(t)=$ $-0.1[\sin (t), \cos (t), \cos (t)]^{T}$ for 3 -DOF robots. The control parameters are selected and shown in Table 1, which guaranteed the establishment of (13) and (22).

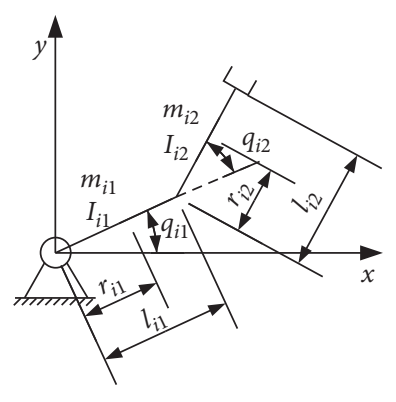

(a)

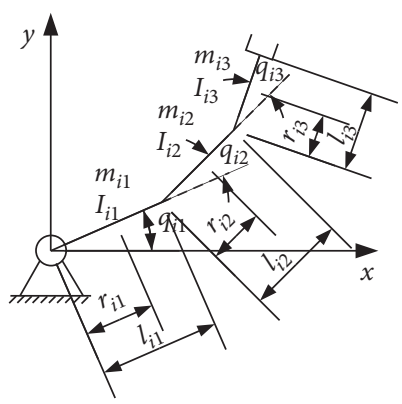

(b)
Figure 1: Mechanical structures of 2-DOF (a) and 3-DOF (b) robots.

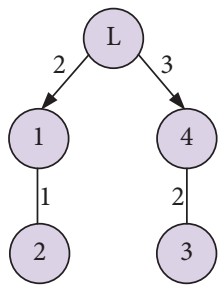

(a)

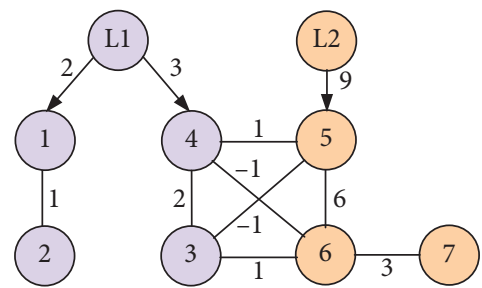

(b)
Figure 2: Interaction topologies of Example 1 (a) and Example 2 (b).

TABLE 1: Control parameters of EBHC algorithms I and II.

\begin{tabular}{lccccccccc}
\hline Control parameters & $\alpha$ & $\beta$ & $\rho_{1}$ & $\rho_{2}$ & $\omega_{i}$ & $\kappa_{o i}$ & $\kappa_{s i}$ & $\kappa_{d i}$ & $\Lambda_{i}$ \\
\hline 2-DOF & 8 & 12 & 0.5 & $2 / 3$ & 0.8 & $10 I_{2}$ & $12 I_{2}$ & $15 I_{2}$ & $5 I_{5}$ \\
3-DOF & 8 & 12 & 0.5 & $2 / 3$ & 0.8 & $10 I_{2}$ & $15 I_{3}$ & $12 I_{3}$ & $8 I_{9}$
\end{tabular}

Besides, the virtual leader is chosen as

$$
\left\{\begin{array}{l}
x_{r}=[7+2.5 \sin (0.4 t), 4+2.5 \cos (0.4 t)]^{T}, \\
v_{r}=[2.5 \times 0.4 \cos (0.4 t),-2.5 \times 0.4 \sin (0.4 t)]^{T}, \\
a_{r}=\left[-2.5 \times 0.4^{2} \sin (0.4 t),-2.5 \times 0.4^{2} \cos (0.4 t)\right]^{T},
\end{array}\right.
$$

which makes Assumption 2 hold. The NHRS is required to form a time-varying square and move along the trajectory of the leader with the leader being its centre, where the timevarying square is specified by

$$
\left\{\begin{array}{l}
h_{i}=\left[0.4 \cos \left(\frac{(2 i-1) \pi}{4}+t\right), 0.4 \sin \left(\frac{(2 i-1) \pi}{4}+t\right)\right]^{T}, \\
\dot{h}_{i}=\left[-0.4 \sin \left(\frac{(2 i-1) \pi}{4}+t\right), 0.4 \cos \left(\frac{(2 i-1) \pi}{4}+t\right)\right]^{T}, \\
\ddot{h_{i}}=\left[-0.4 \cos \left(\frac{(2 i-1) \pi}{4}+t\right),-0.4 \sin \left(\frac{(2 i-1) \pi}{4}+t\right)\right]^{T},
\end{array}\right.
$$

for $i \in\{1,2,3,4\}$. On the contrary, the elements of the initial values $\widehat{x}_{i}(0), \dot{\hat{x}}_{i}(0), q_{i}(0), \dot{q}_{i}(0)$, and $\widehat{\vartheta}_{i}(0)$ are randomly chosen in $[0,15],[-1,1],[-20,20],[-5,5]$, and $[-2,2]$, respectively. 


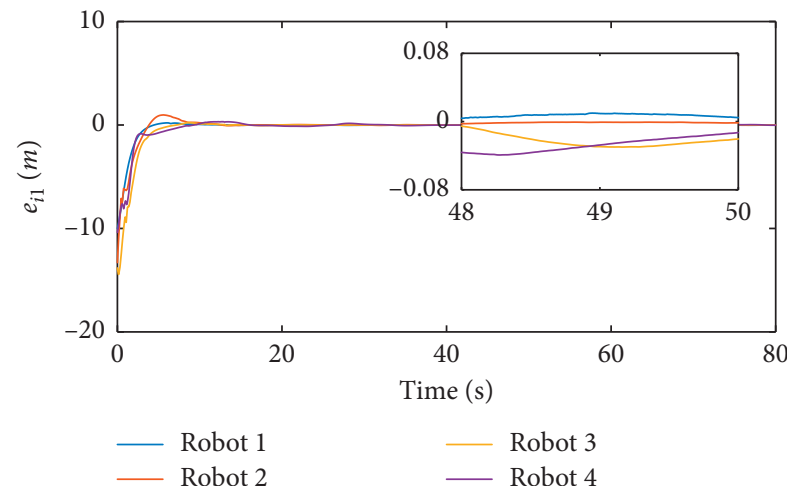

(a)

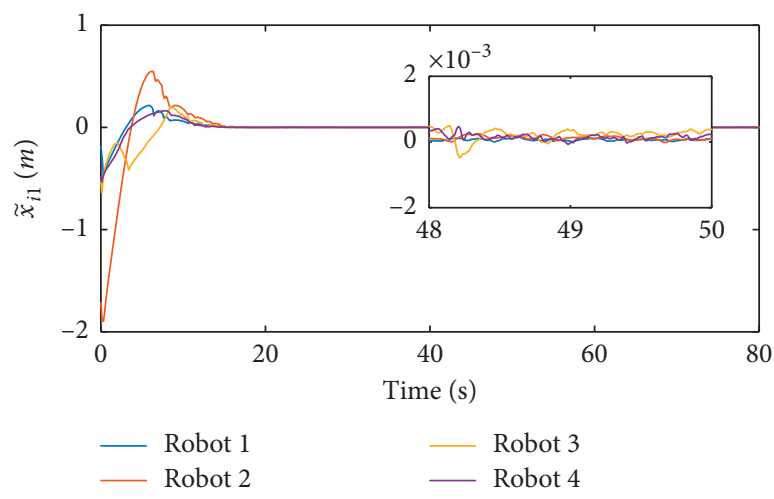

(c)

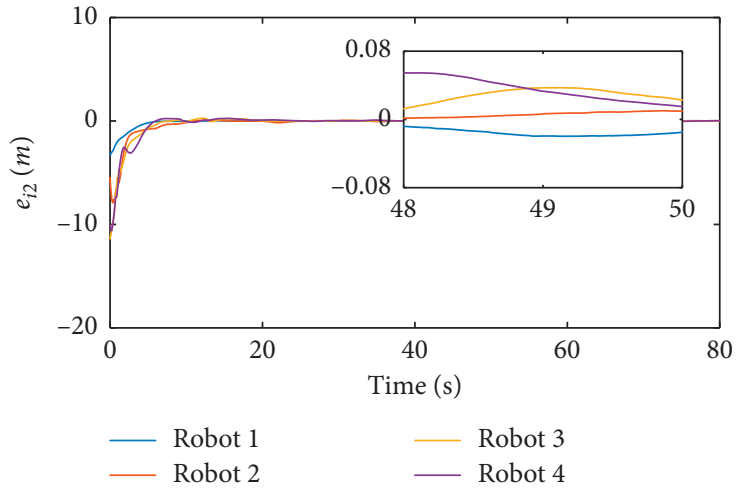

(b)

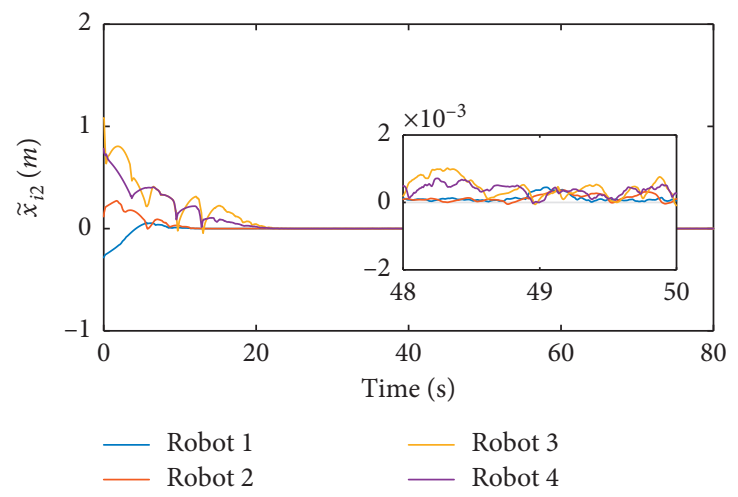

(d)

FIgURE 3: Evolution of $e_{i 1}$ (a) $e_{i 2}$ (b) $\tilde{x}_{i 1}$ (c), and $\tilde{x}_{i 2}$ (d) in Example 1.

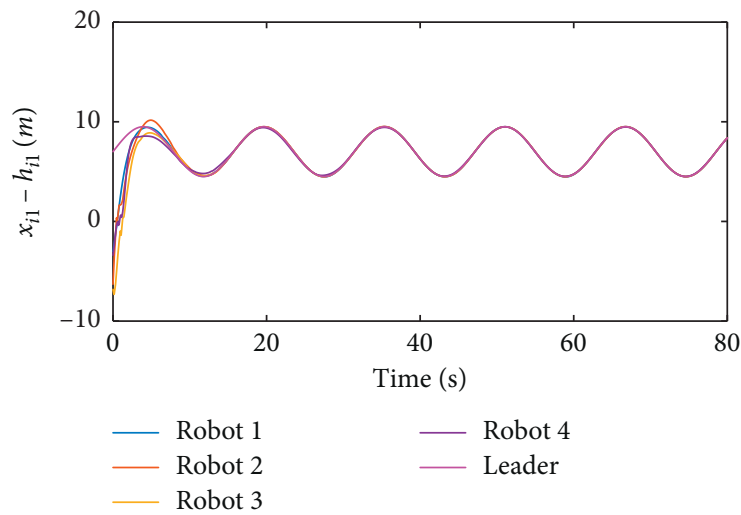

(a)

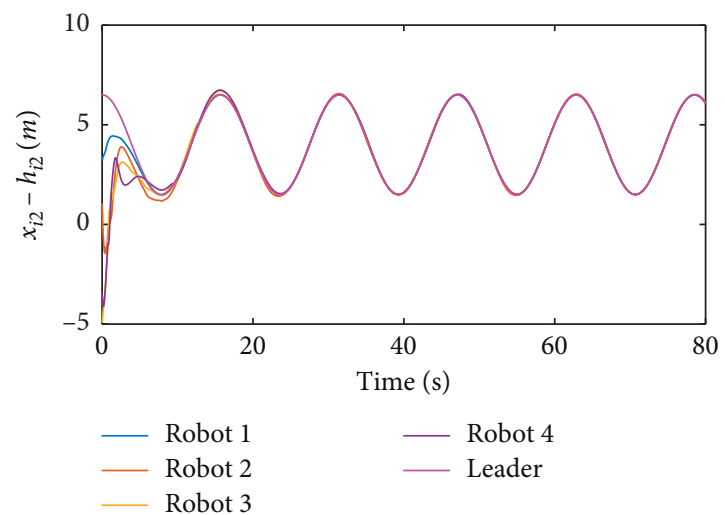

(b)

Figure 4: Continued. 


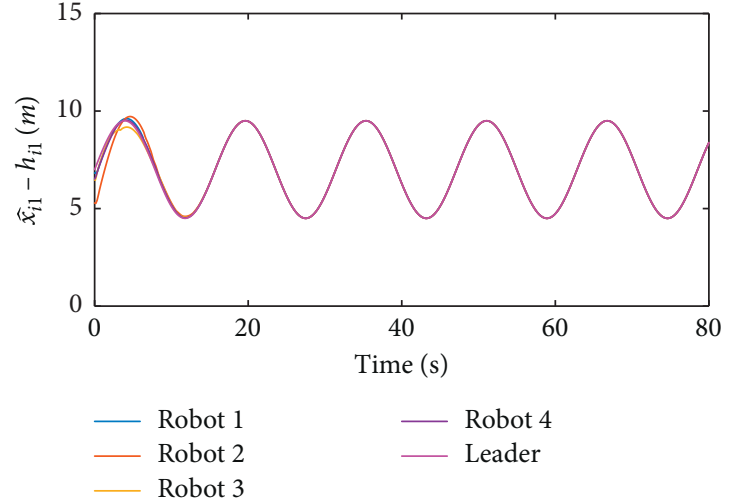

(c)

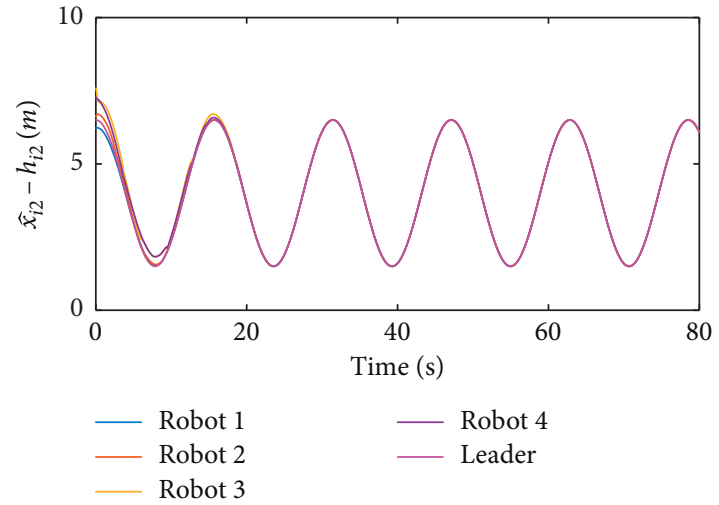

(d)

FIGURE 4: Evolution of $x_{i 1}-h_{i 1}$ (a), $x_{i 2}-h_{i 2}$ (b), $\hat{x}_{i 1}-h_{i 1}$ (c), and $\hat{x}_{i 2}-h_{i 2}$ (d) in Example 1.

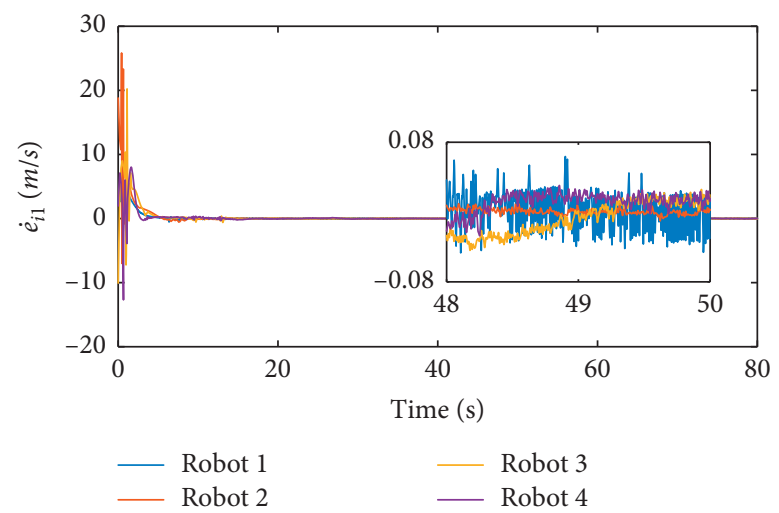

(a)

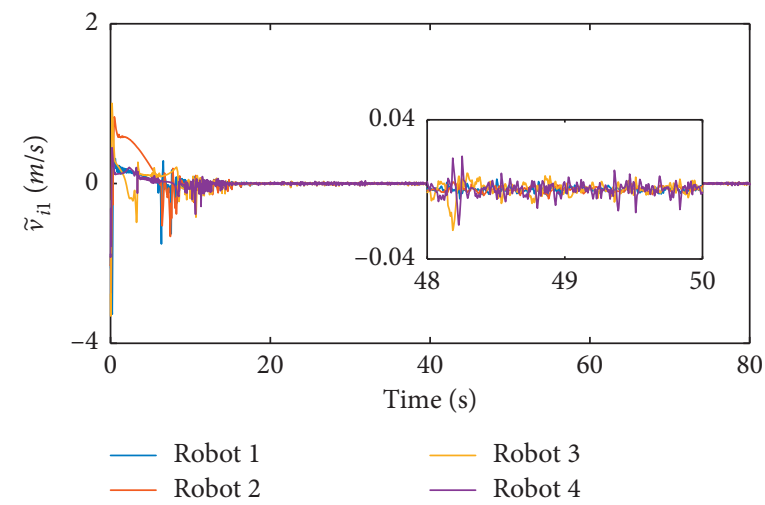

(c)

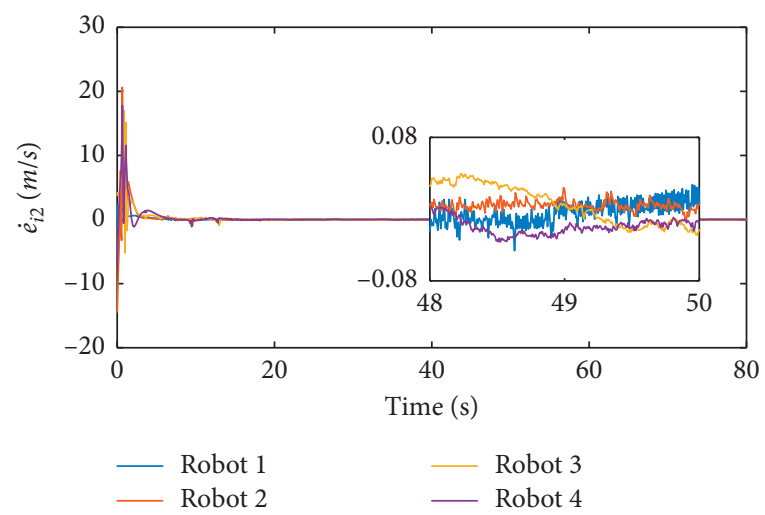

(b)

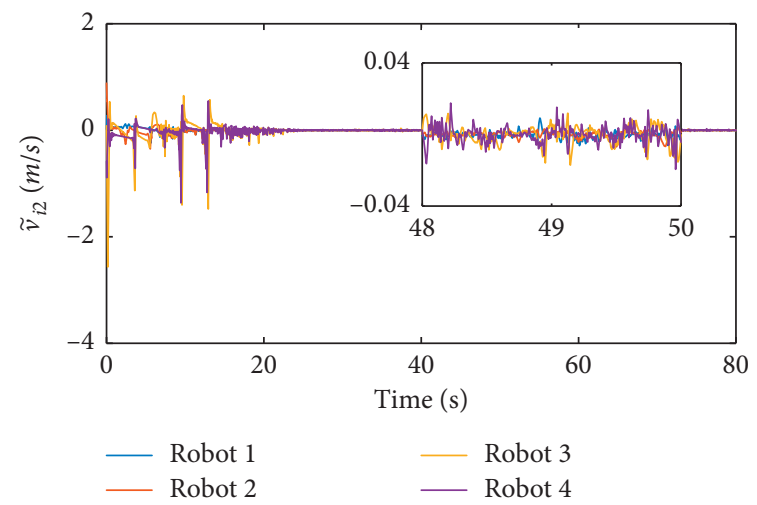

(d)

FIGURE 5: Evolution of $\dot{e}_{i 1}(\mathrm{a}), \dot{e}_{i 2}(\mathrm{~b}), \widetilde{v}_{i 1}(\mathrm{c})$, and $\widetilde{v}_{i 2}$ (d) in Example 1.

The simulation results of Example 1 are shown in Figures 3-8. For details, from Figures 3(c), 3(d), 4(c), and 4(d), it can be observed that the estimators $\widehat{x}_{i}, i \in\{1,2,3,4\}$, converge to $x_{r}+h_{i}$ almost when $t \geq 25.5 \mathrm{~s}$. Also, Figures 3(a), 3(b), 4(a), and 4(b) imply that the task-space coordinates of each robot can asymptotically track the trajectory of $x_{r}+h_{i}$. Similarly, Figures 5 and 6 indicate the tracking performance of $v_{i}$ and $\widehat{v}_{i}$. Figure 7 provides the trajectories of all the robots inside and the leader, from which we can observe that the four robots form a square, and the square is rotating itself with a certain angular velocity while moving along the trajectory of the leader. It is noted that the tracking errors $e_{i}$ and $\dot{e}_{i}$ are bounded by $[-0.08,0.08]$, which are tiny enough to support our main results. 


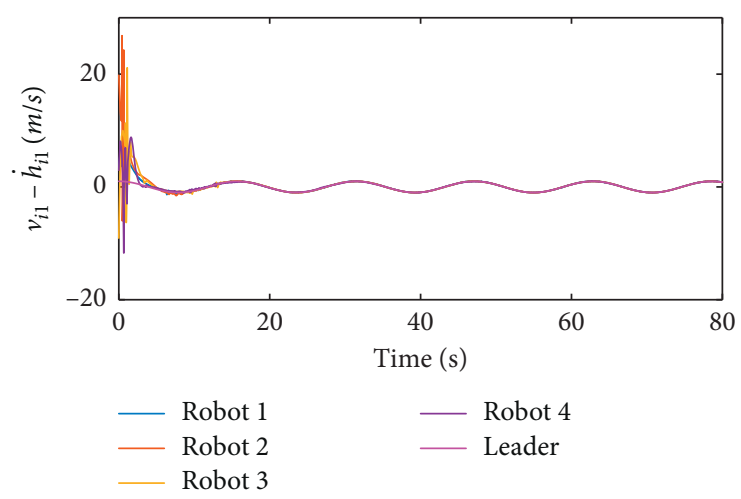

(a)

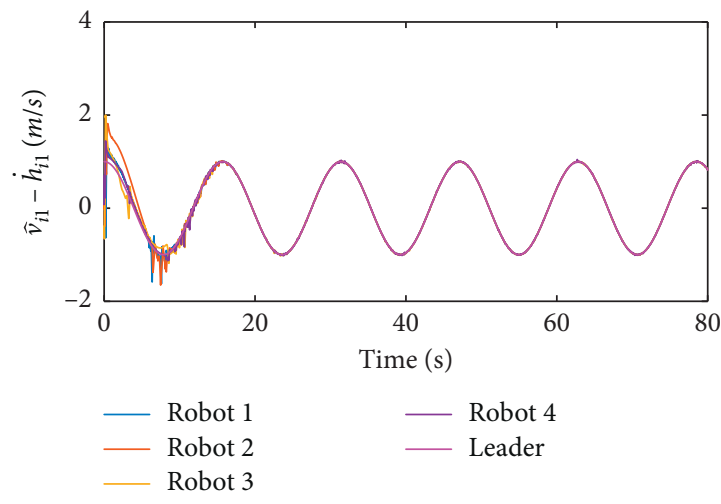

(c)

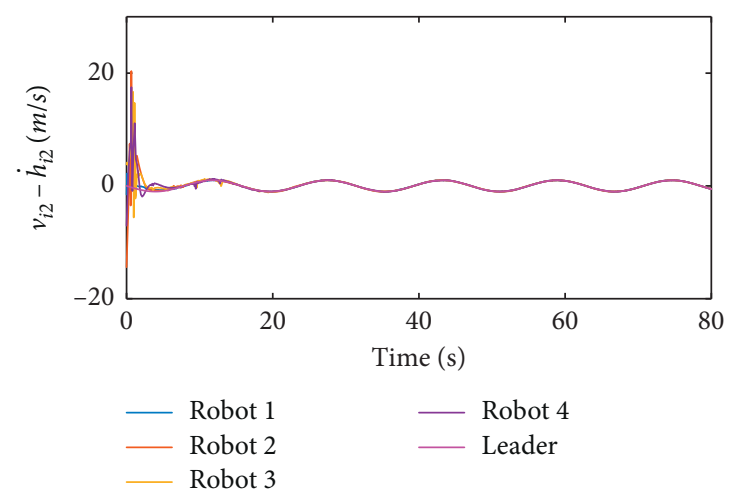

(b)

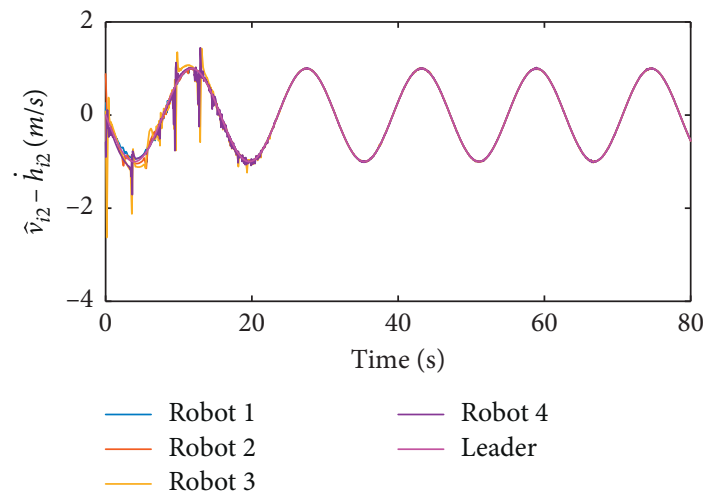

(d)

FIGURE 6: Evolution of $v_{i 1}-\dot{h}_{i 1}(\mathrm{a}), v_{i 2}-\dot{h}_{i 2}(\mathrm{~b}), \widehat{v}_{i 1}-\dot{h}_{i 1}$ (c), and $\widehat{v}_{i 2}-\dot{h}_{i 2}$ (d) in Example 1.

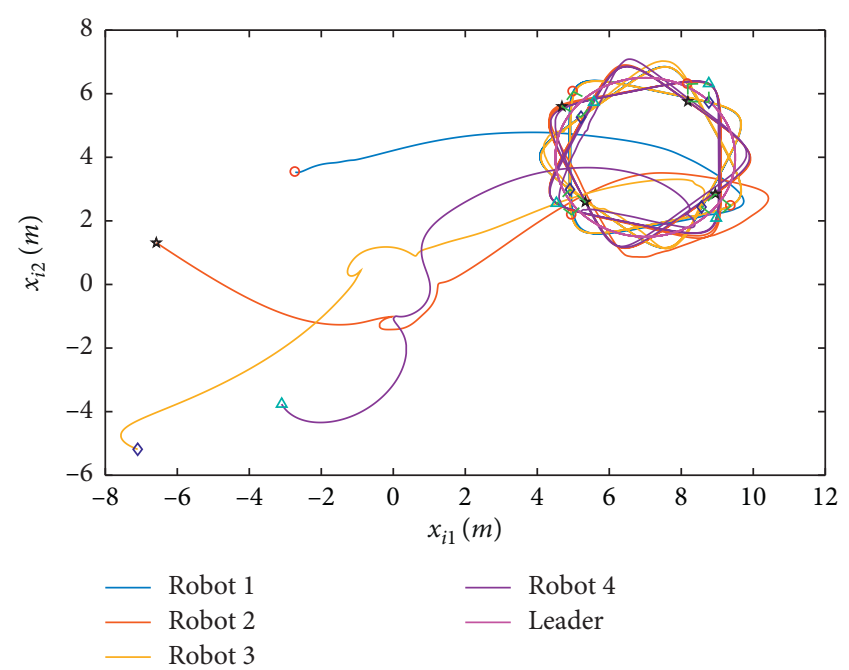

FIgURE 7: Task-space time-varying formation tracking performance of the NHRS shown in the XY plane in Example 1.

On the contrary, we choose (redundant) robot 1 to verify that the subtask can also be finished but does not conflict with the tracking task. The negative gradient of the performance index corresponding to robot 1 is designed as

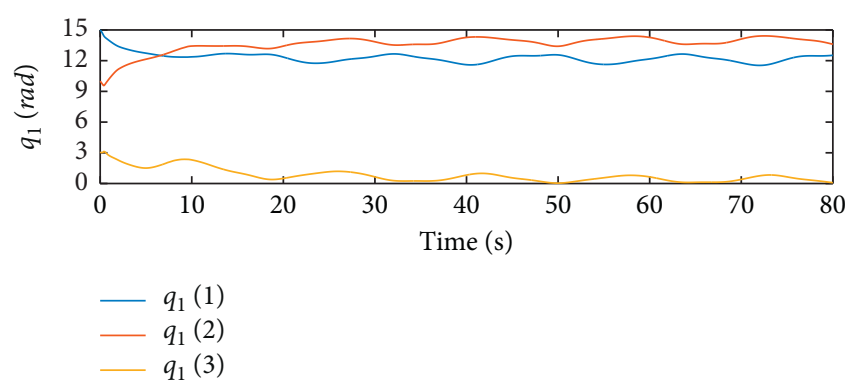

(a)

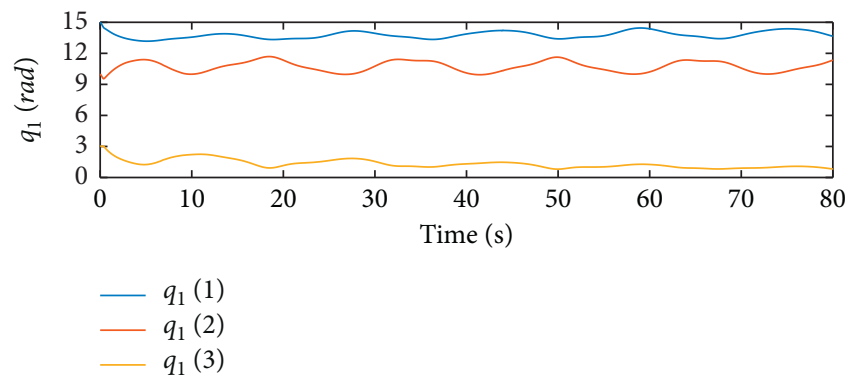

(b)

FIgURE 8: Joint-space coordinates of robot 1 with (a) and without (b) subtask control. 


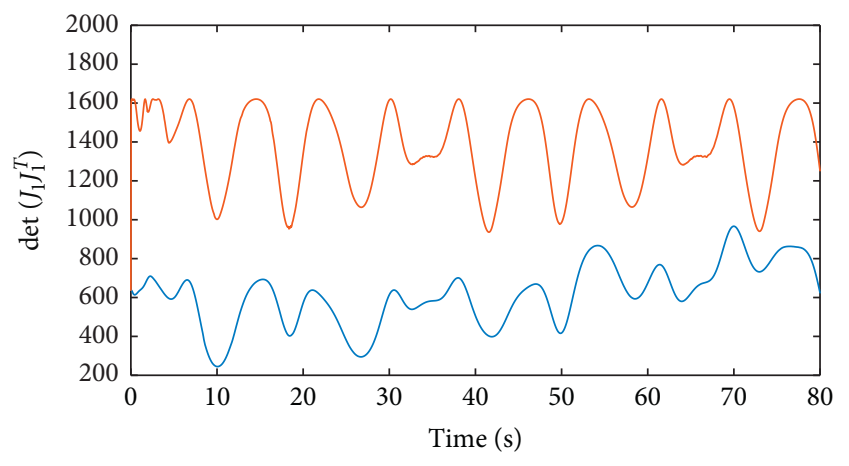

— Without singularity avoidance control
— With singularity avoidance control

FIGURE 9: Manipulability of robot 1 with (a) and without (b) singularity avoidance control.

$\varphi_{1}=\operatorname{col}\left(0.8\left(12-q_{1}(1)\right), 0,0\right)$, where $q_{1}(1)$ denotes the first joint of robot 1 . The designed $\varphi_{1}$ aims at forcing the first joint of robot 1 toward $12 \mathrm{rad}$. It is clearly shown in Figure 8 that $q_{1}(1)$ is forced to $12 \mathrm{rad}$ compared with the case that no subtask control is involved. Next, we choose $\varphi_{1}=\partial / \partial q_{1}\left(\operatorname{det}\left(J_{1} J_{1}^{T}\right)\right)$ to verify the singularity avoidance method presented in Remark 4. It can be observed in Figure 9 that the manipulability of robot 1 is enhanced with subtask control. It thus can be obtained that both the tracking task and the subtask can be addressed under the proposed EBHC algorithm I.

Example 2. EBHC algorithm II (31) and (32) are employed to address the multiple time-varying formation tracking problem of the NHRS consisting of seven robots in the case of two virtual leaders, among which robots 1 and 7 are redundant and the remain five robots are nonredundant. The NHRS considered here can be divided into two subnetworks, where robots $1-4$ and robots 5-7 belong to the first and the second subnetwork, respectively. The physical parameters of robots 1 are the same as in Example 1, and choose $m_{i}=\operatorname{col}(3.8+0.1 i, 3.3+0.1 i) \mathrm{kg}$, $l_{i}=\operatorname{col}(6.6+0.2 i, 5.6+0.2 i) \mathrm{m}, \quad$ and $\quad r_{i}=0.5 l_{i}$ for $i \in\{2,3,4,5,6\}, \quad$ and $\quad m_{7}=\operatorname{col}(3.2,2.2,1.2) \mathrm{kg}$, $l_{7}=\operatorname{col}(5.4,4.4,3.4) \mathrm{m}$, and $r_{7}=0.5 l_{1}$. The interactions among the robots and the leaders are depicted in Figure 2(b), which implies that the pinning matrix $\mathscr{B}$ and the Laplacian matrix $\mathscr{L}$ are given as $\mathscr{B}=\operatorname{diag}(2,0,0,3,9,0,0)$ and

$$
\mathscr{L}=\left(\begin{array}{ccccccc}
1 & -1 & 0 & 0 & 0 & 0 & 0 \\
-1 & 1 & 0 & 0 & 0 & 0 & 0 \\
0 & 0 & 2 & -2 & 1 & -1 & 0 \\
0 & 0 & -2 & 2 & -1 & 1 & 0 \\
0 & 0 & 1 & -1 & 6 & -6 & 0 \\
0 & 0 & -1 & 1 & -6 & 9 & -3 \\
0 & 0 & 0 & 0 & 0 & -3 & 3
\end{array}\right) .
$$

The external disturbances, the control parameters, leader 1 , and the formation offsets of robots $1-4$ are the same as in Example 1. Besides, leader 2 is described as

$$
\left\{\begin{array}{l}
x_{r, 2}=[3.5 \cos (0.5 t), 8+2.5 \sin (0.5 t)]^{T}, \\
v_{r, 2}=[-3.5 \times 0.5 \sin (0.5 t), 2.5 \times 0.5 \cos (0.5 t)]^{T}, \\
a_{r, 2}=\left[-3.5 \times 0.5^{2} \cos (0.5 t),-2.5 \times 0.5^{2} \sin (0.5 t)\right]^{T}
\end{array}\right.
$$

The second subnetwork is expected to form a timevarying regular triangle, which is specified by

$$
\begin{aligned}
& \left\{\begin{array}{l}
h_{5}=0.1[0,6+2 \sin t]^{T}, \\
\dot{h}_{5}=0.1[0,2 \cos t]^{T}, \\
\ddot{h}_{5}=0.1[0,-2 \sin t]^{T},
\end{array}\right. \\
& \left\{\begin{array}{l}
h_{6}=0.1[-3 \sqrt{3}-\sqrt{3} \sin t,-3-\sin t]^{T}, \\
\dot{h}_{6}=0.1[-\sqrt{3} \cos t,-\cos t]^{T}, \\
\ddot{h_{6}}=0.1[\sqrt{3} \sin t, \sin t]^{T},
\end{array}\right. \\
& \left\{\begin{array}{l}
h_{7}=0.1[3 \sqrt{3}+\sqrt{3} \sin t,-3-\sin t]^{T}, \\
\dot{h}_{7}=0.1[\sqrt{3} \cos t,-\cos t]^{T}, \\
\ddot{h}_{7}=0.1[-\sqrt{3} \sin t, \sin t]^{T} .
\end{array}\right.
\end{aligned}
$$

Finally, the initial values are randomly chosen in the same corresponding ranges given in Example 1.

The simulation results are shown in Figures 10-14. Similarly, Figures 10 and 11 indicate that $x_{i}$ can eventually track the desired position, and the convergence of $\hat{x}_{i}-h_{i}$ is completed in finite time. Besides, Figure 12 depicts the tracking performance of $v_{i}$ and $\widehat{v}_{i}$. Figure 13 indicates that the NHRS can achieve the multiple time-varying formation in the $X Y$ plane under the proposed EBHC algorithm II, which is also depicted in Figure 14. In detail, the robots belonging to the first subnetwork form the time-varying square as same as in Example 1, and the robots belonging to the second subnetwork form a time-varying regular triangle with its size changing periodically. Note that the tracking errors in Figures 10 and 12 do not converge to zero. This is mainly because although it has been theoretically proved that the states converge to zero asymptotically, the final simulation results converge to the neighbourhood of the origin due to the presence of external disturbances, 

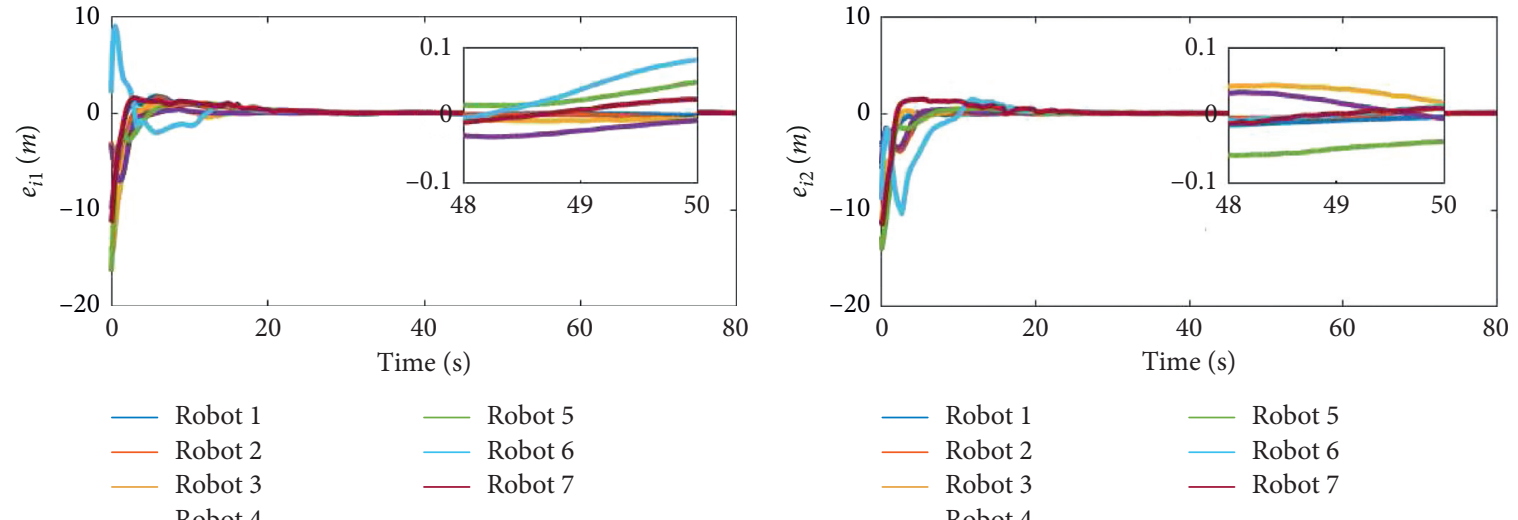

(a)

(b)

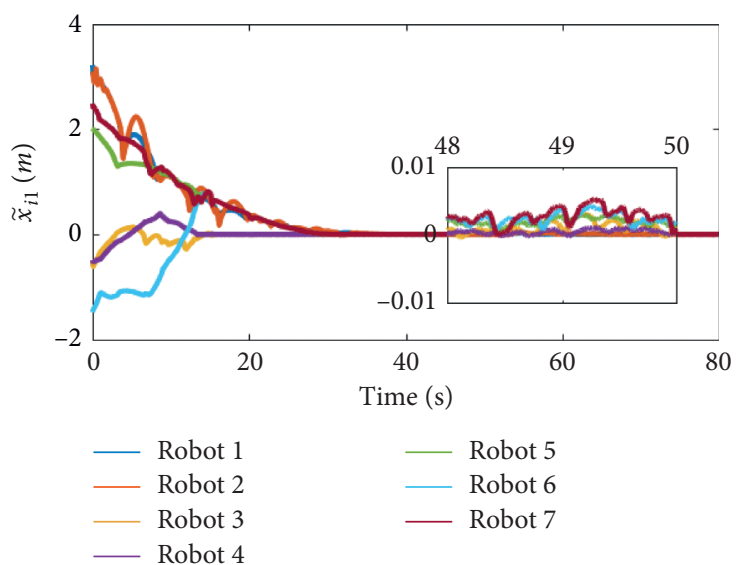

(c)

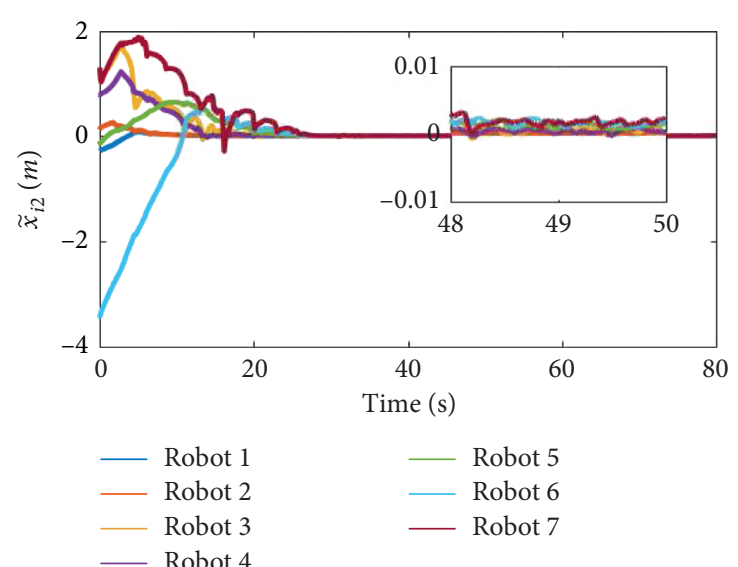

(d)

Figure 10: Evolution of $e_{i 1}(\mathrm{a}), e_{i 2}(\mathrm{~b}), \tilde{x}_{i 1}(\mathrm{c})$, and $\tilde{x}_{i 2}$ (d) in Example 2.

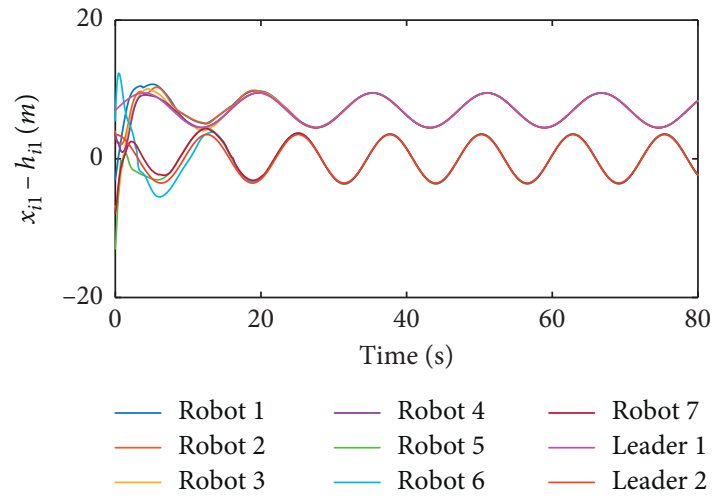

(a)

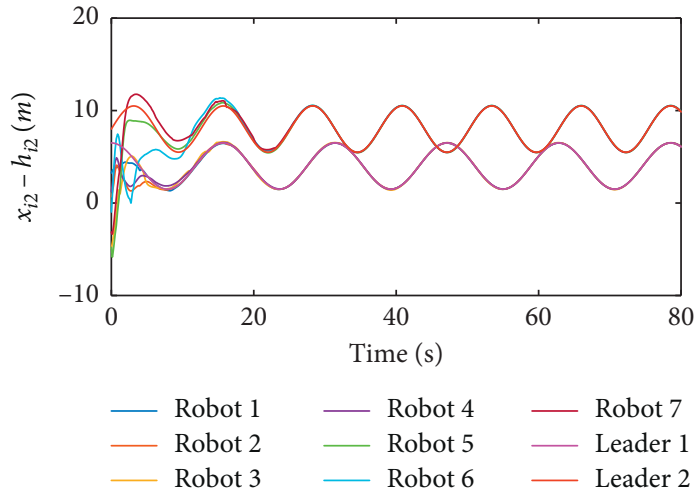

(b)

Figure 11: Continued. 


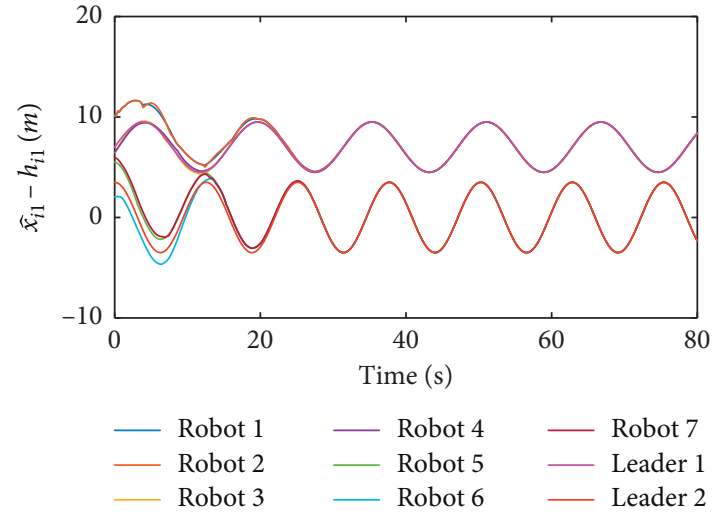

(c)

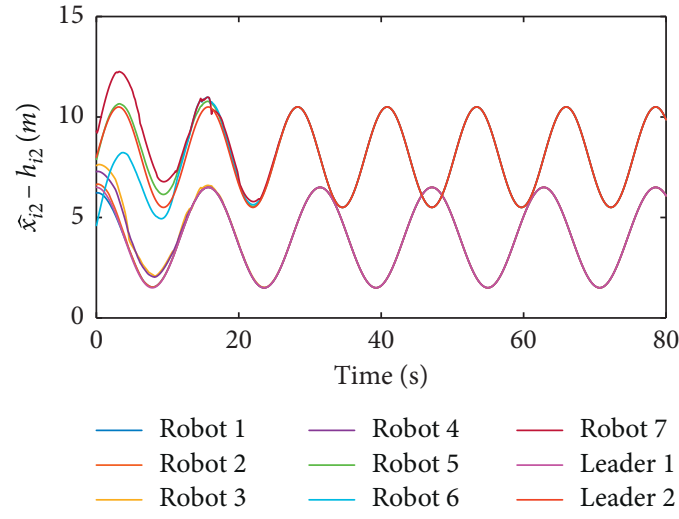

(d)

FIGURE 11: Evolution of $x_{i 1}-h_{i 1}(t)(\mathrm{a}), x_{i 2}-h_{i 2}(t)(\mathrm{b}), \widehat{x}_{i 1}-h_{i 1}$ (c), and $\widehat{x}_{i 2}-h_{i 2}$ (d) in Example 2.

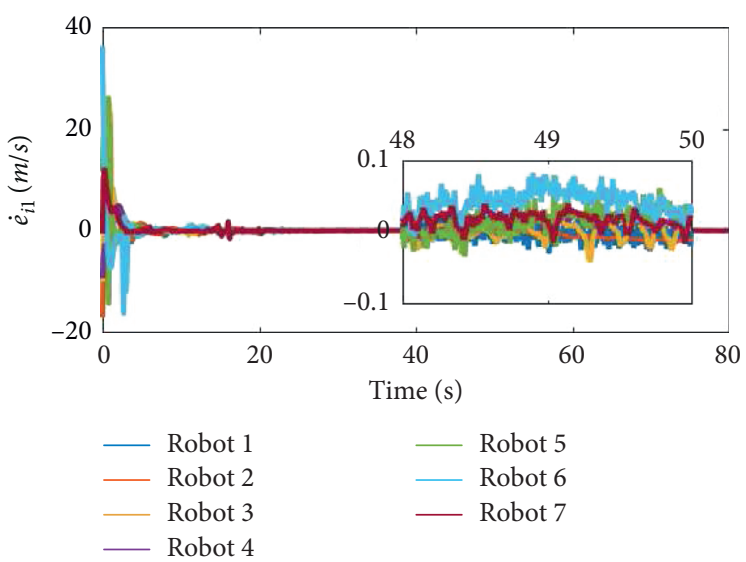

(a)

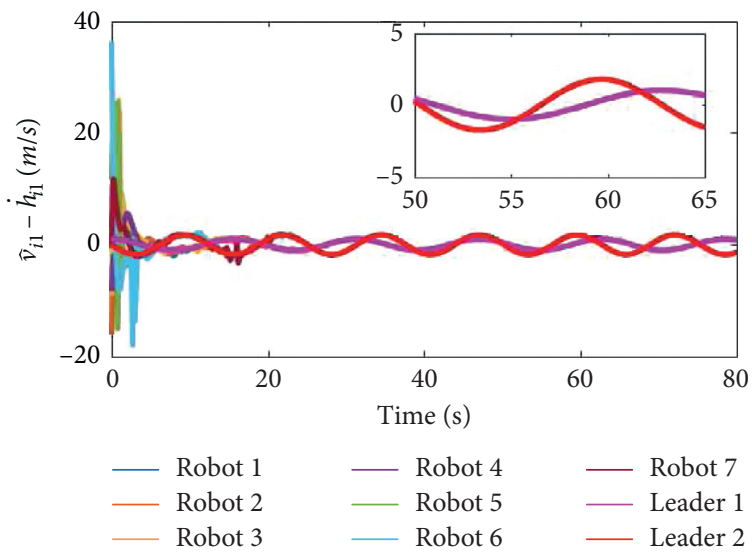

(c)

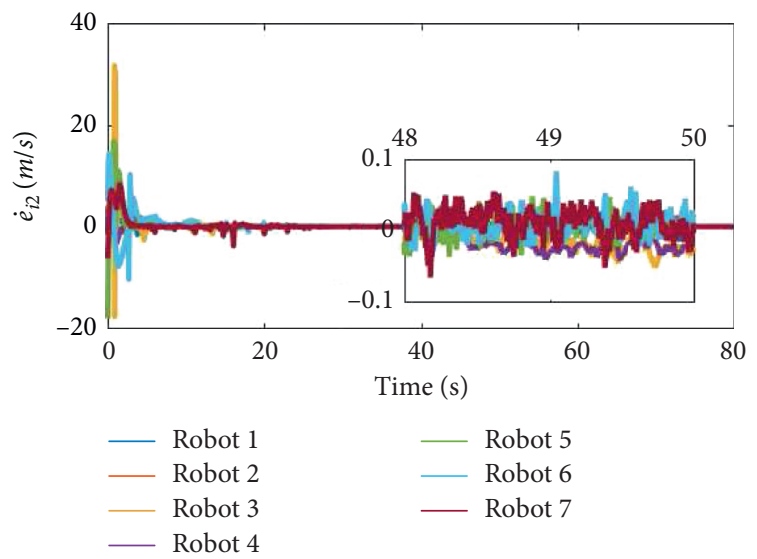

(b)

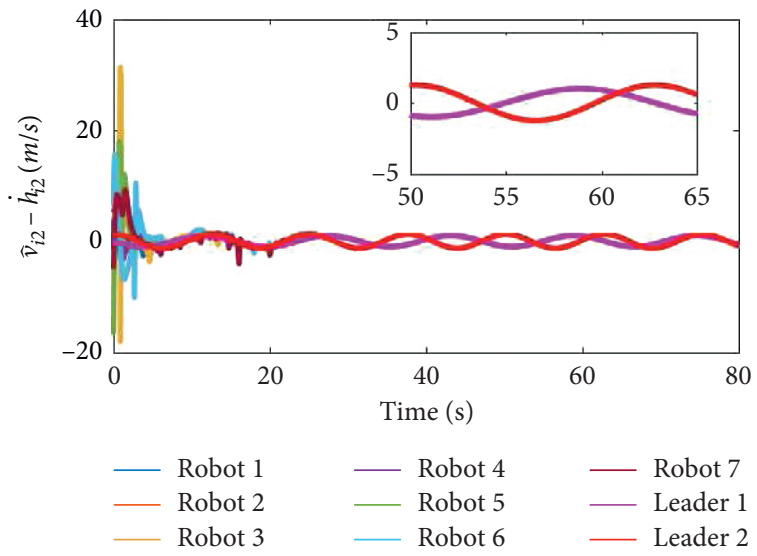

(d)

FIGURE 12: Evolution of $\dot{e}_{i 1}(\mathrm{a}), \dot{e}_{i 2}(\mathrm{~b}), v_{i 1}-\dot{h}_{i 1}(t)(\mathrm{c})$, and $v_{i 2}-\dot{h}_{i 2}(t)(\mathrm{d})$ in Example 2.

parametric uncertainties, and kinematic redundancy and the employment of the sign function. This phenomenon is very general in the applications of the sliding mode control technique on regulating complex systems with various uncertainties. Thus, it is theoretically correct to obtain the simulation results shown in Figures 10 and 12 . It thus can be concluded that the time-varying formation tracking problems of NHRSs with both a single leader and multiple leaders can be solved under the proposed EBHC algorithms. 


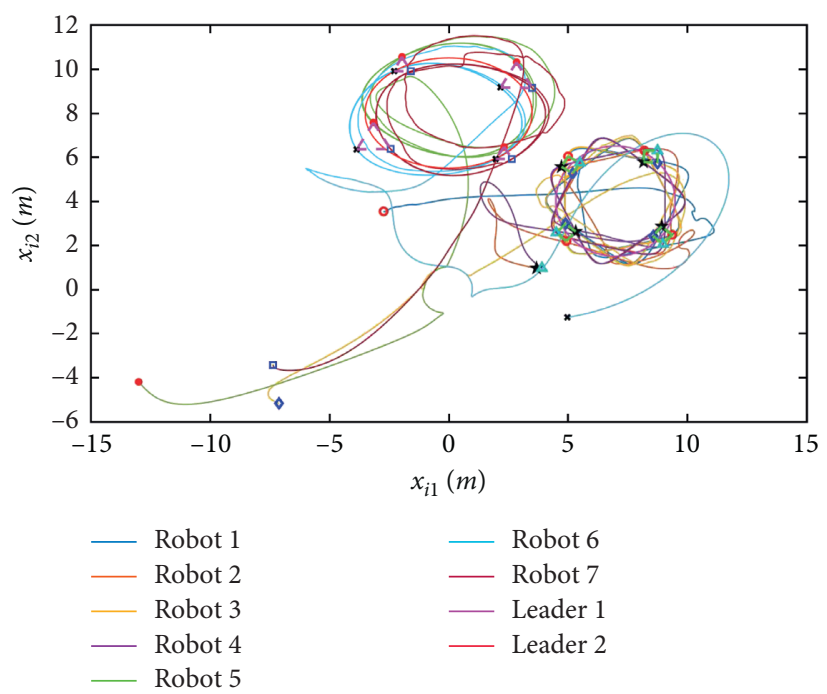

FIGURE 13: Task-space time-varying formation tracking performance of the NHRS shown in the XY plane in Example 2.

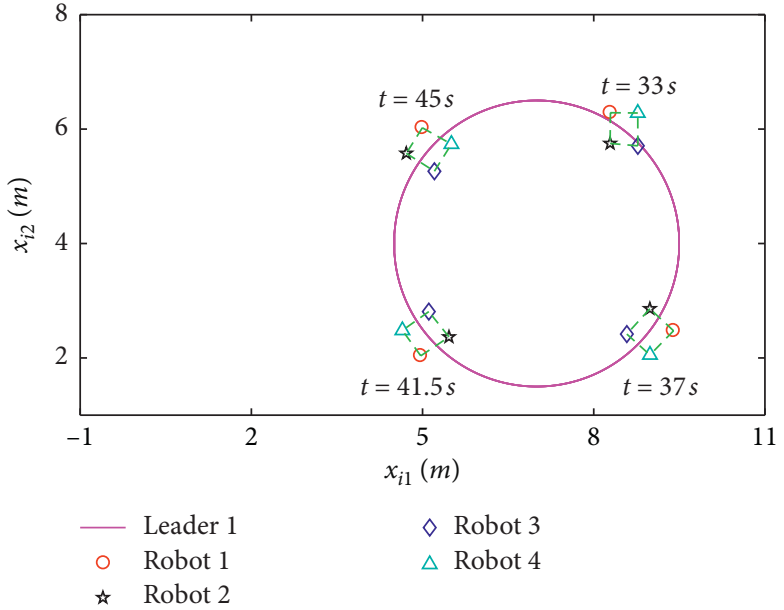

(a)

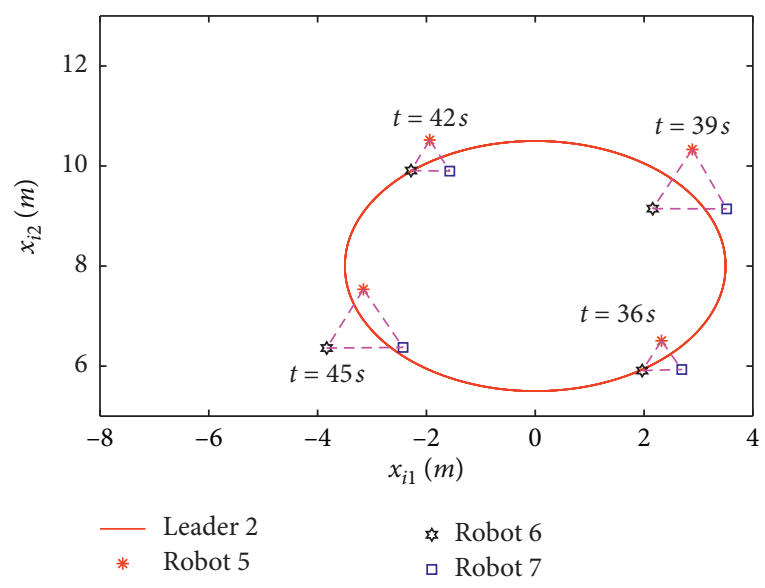

(b)

FIGURE 14: Tracking trajectories of the first subnetwork (a) and the second subnetwork (b) in the XY plane.

\section{Conclusion}

This paper mainly focused on the time-varying formation tracking problem of NHRSs in the task space considering the parameter uncertainties and external disturbances. Two novel EBHC algorithms, where both redundant robots and nonredundant robots have been taken into account, have been proposed to realize the time-varying formation in the case of a single virtual leader and multiple leaders, respectively. Besides, the designed distributed estimator algorithms are able to guarantee the availability of the corresponding leaders' information for each robot in finite time. The sufficient conditions have been derived by invoking Lyapunov stability and input-to-state stability. The presented simulation results have given satisfactory performance of proposed EBHC algorithms. Future works will focus on the time-varying formation tracking problem of NHRSs with time delays.

\section{Data Availability}

This paper mainly brings some theoretical perspectives and methodologic approaches. All the figures are obtained through simulations based on the presented approaches. No data were used to support this study.

\section{Conflicts of Interest}

The authors declare that there are no conflicts of interest regarding the publication of this paper.

\section{Acknowledgments}

This work was supported by the National Natural Science Foundation of China $(61703374,61971181,61973110$, 61603127, and 61503282), the Teaching Laboratory Open Fund Project of the China University of Geosciences 
(Wuhan) (1910491B05), the Fundamental Research Funds for the Central Universities, China University of Geosciences (Wuhan) (CUG170656), the Natural Science Foundation of Hubei Province of China (2019CFB559 and 2016CFB514), and the Research Fund for the Doctoral Program of the Hubei University of Technology (BSQD2015044).

\section{References}

[1] R. Olfati-Saber and R. M. Murray, "Consensus problems in networks of agents with switching topology and time-delays," IEEE Transactions on Automatic Control, vol. 49, no. 9, pp. 1520-1533, 2004.

[2] L. Wang, M.-F. Ge, Z. Zeng, and J. Hu, "Finite-time robust consensus of nonlinear disturbed multiagent systems via twolayer event-triggered control," Information Sciences, vol. 466, pp. 270-283, 2018.

[3] M. F. Ge, Z. W. Liu, G. Wen, X. Yu, and T. Huang, "Hierarchical controller-estimator for coordination of networked Euler-Lagrange systems," IEEE Transactions on Cybernetics, 2019, doi: 10.1109/tcyb.2019.2914861.

[4] Z. W. Liu, G. Wen, X. Yu, Z. H. Guan, and T. Huang, "Delayed impulsive control for consensus of multi-agent systems with switching communication graphs," IEEE Transactions on Cybernetics, 2019, doi: 10.1109/tcyb.2019.2926115.

[5] X. Hu, Z. W. Liu, G. Wen, X. Yu, and C. Li, "Branch-wise parallel successive algorithm for online voltage regulation in distribution networks," IEEE Transactions on Smart Grid, vol. 10, no. 6, pp. 6678-6689, 2019.

[6] M.-F. Ge, Z.-H. Guan, B. Hu, D.-X. He, and R.-Q. Liao, "Distributed controller-estimator for target tracking of networked robotic systems under sampled interaction," Automatica, vol. 69, pp. 410-417, 2016.

[7] C.-D. Liang, L. Wang, X.-Y. Yao, Z.-W. Liu, and M.-F. Ge, "Multi-target tracking of networked heterogeneous collaborative robots in task space," Nonlinear Dynamics, vol. 97, no. 2, pp. 1159-1173, 2019.

[8] G. Wen, T. Huang, W. Yu, Y. Xia, and Z. W. Liu, "Cooperative tracking of networked agents with a high-dimensional leader: qualitative analysis and performance evaluation," IEEE Transactions on Cybernetics, vol. 48, no. 7, pp. 2060-2073, 2017.

[9] X.-W. Jiang, C.-Y. Chen, Q.-S. Yang, X.-J. Qu, and H.-C. Yan, "Optimal tracking performance for SIMO systems with packet dropouts and control energy constraints," IET Control Theory \& Applications, vol. 12, no. 12, pp. 1714-1721, 2018.

[10] X.-S. Zhan, L.-L. Cheng, J. Wu, Q.-S. Yang, and T. Han, "Optimal modified performance of MIMO networked control systems with multi-parameter constraints," ISA Transactions, vol. 84, pp. 111-117, 2019.

[11] X.-S. Zhan, J. Wu, T. Jiang, and X.-W. Jiang, "Optimal performance of networked control systems under the packet dropouts and channel noise," ISA Transactions, vol. 58, pp. 214-221, 2015.

[12] C. Y. Chen, W. Gui, L. Wu, Z. Liu, and H. Yan, "Tracking performance limitations of MIMO networked control systems with multiple communication constraints," IEEE Transactions on Cybernetics, 2019, doi: 10.1109/tcyb.2019.2912973.

[13] J.-W. Hu, X.-S. Zhan, J. Wu, and H.-C. Yan, "Optimal tracking performance of NCSs with time-delay and encodingdecoding constraints," International Journal of Control, Automation and Systems, vol. 18, no. 4, 2020, doi: 10.1007/ s12555-019-0300-5.
[14] T. F. Ding, M. F. Ge, Z. W. Liu, Y. W. Wang, and H. R. Karimi, "Discrete-Communication-based bipartite tracking of networked robotic systems via hierarchical hybrid control," IEEE Transactions on Circuits and Systems I: Regular Papers, vol. 67, no. 4, 2020, doi: 10.1109/tcsi.2019.2961804.

[15] J. Wu, Q. Deng, C. Y. Chen, and H. Yan, "Bipartite consensus for second order multi-agent systems with exogenous disturbance via pinning control," IEEE Access, vol. 7, pp. 186563-186571, 2019.

[16] C. D. Liang, M. F. Ge, Z. W. Liu, Y. W. Wang, and H. R. Karimi, "Output multi-formation tracking of networked heterogeneous robotic systems via finite-time hierarchical control," IEEE Transactions on Cybernetics, 2020, doi: 10.1109/tcyb.2020.2968403.

[17] T. Han, Z.-H. Guan, M. Chi, B. Hu, T. Li, and X.-H. Zhang, "Multi-formation control of nonlinear leader-following multiagent systems," ISA Transactions, vol. 69, pp. 140-147, 2017.

[18] X. Dong, Y. Zhou, Z. Ren, and Y. Zhong, “Time-varying formation tracking for second-order multi-agent systems subjected to switching topologies with application to quadrotor formation flying," IEEE Transactions on Industrial Electronics, vol. 64, no. 6, pp. 5014-5024, 2016.

[19] X. Dong and G. Hu, "Time-varying formation tracking for linear multiagent systems with multiple leaders," IEEE Transactions on Automatic Control, vol. 62, no. 7, pp. 3658-3664, 2017.

[20] X.-Y. Yao, H.-F. Ding, and M.-F. Ge, "Formation-containment control for multi-robot systems with two-layer leaders via hierarchical controller-estimator algorithms," Journal of the Franklin Institute, vol. 355, no. 12, pp. 5272-5290, 2018.

[21] Y.-W. Wang, X.-K. Liu, J.-W. Xiao, and Y. Shen, "Output formation-containment of interacted heterogeneous linear systems by distributed hybrid active control," Automatica, vol. 93, pp. 26-32, 2018.

[22] Y.-W. Wang, X.-K. Liu, J.-W. Xiao, and X. Lin, "Output formation-containment of coupled heterogeneous linear systems under intermittent communication," Journal of the Franklin Institute, vol. 354, no. 1, pp. 392-414, 2017.

[23] L. Wang, Z. Zeng, J. Hu, and X. Wang, "Controller design for global fixed-time synchronization of delayed neural networks with discontinuous activations," Neural Networks, vol. 87, pp. 122-131, 2017.

[24] L. Wang, Y. Shen, Q. Yin, and G. Zhang, "Adaptive synchronization of memristor-based neural networks with timevarying delays," IEEE Transactions on Neural Networks and Learning Systems, vol. 26, no. 9, pp. 2033-2042, 2014.

[25] G. Zhang, J. Hu, and Z. Zeng, "New criteria on global stabilization of delayed memristive neural networks with inertial item," IEEE Transactions on Cybernetics, 2019, doi: 10.1109/ TCYB.2018.2889653.

[26] G. Zhang, J. Hu, and F. Jiang, "Exponential stability criteria for delayed second-order memristive neural networks," Neurocomputing, vol. 315, pp. 439-446, 2018.

[27] X. W. Zhao, Z. H. Guan, J. Li, X. H. Zhang, and C. Y. Chen, "Flocking of multi-agent nonholonomic systems with unknown leader dynamics and relative measurements," International Journal of Robust and Nonlinear Control, vol. 27, no. 17, pp. 3685-3702, 2017.

[28] R. W. Beard and T. W. McLain, "Multiple UAV cooperative search under collision avoidance and limited range communication constraints," in Proceedings of the 42nd IEEE International Conference on Decision and Control (IEEE Cat. No. 03CH37475), vol. 1, pp. 25-30, IEEE, Maui, HI, USA, December 2003.

[29] Z. Meng, W. Ren, and Z. You, "Distributed finite-time attitude containment control for multiple rigid bodies," Automatica, vol. 46, no. 12, pp. 2092-2099, 2010. 
[30] H. Wang, "Task-space synchronization of networked robotic systems with uncertain kinematics and dynamics," IEEE Transactions on Automatic Control, vol. 58, no. 12, pp. 3169-3174, 2013.

[31] H. Wang and Y. Xie, "Task-space consensus of networked robotic systems: separation and manipulability," 2017, https:// arxiv.org/abs/1702.06265.

[32] P. Hsu, J. Mauser, and S. Sastry, "Dynamic control of redundant manipulators," Journal of Robotic Systems, vol. 6, no. 2, pp. 133-148, 1989.

[33] C.-L. Liu and Y.-P. Tian, "Formation control of multi-agent systems with heterogeneous communication delays," International Journal of Systems Science, vol. 40, no. 6, pp. 627-636, 2009.

[34] K. K. Oh and H. S. Ahn, "Formation control of mobile agents based on distributed position estimation," IEEE Transactions on Automatic Control, vol. 58, no. 3, pp. 737-742, 2012.

[35] M.-F. Ge, Z.-H. Guan, C. Yang, T. Li, and Y.-W. Wang, "Time-varying formation tracking of multiple manipulators via distributed finite-time control," Neurocomputing, vol. 202, pp. 20-26, 2016.

[36] G.-S. Han, Z.-H. Guan, J. Chen, D.-X. He, and M. Chi, "Multitracking of first order multi-agent networks via self-triggered control," Asian Journal of Control, vol. 17, no. 4, pp. 1320-1329, 2015.

[37] G.-S. Han, Z.-H. Guan, J. Li, D.-X. He, and D.-F. Zheng, "Multi-tracking of second-order multi-agent systems using impulsive control," Nonlinear Dynamics, vol. 84, no. 3, pp. 1771-1781, 2016.

[38] Y. Xu, H. Y. Shum, T. Kanade, and J. J. Lee, "Parameterization and adaptive control of space robot systems," IEEE Transactions on Aerospace and Electronic Systems, vol. 30, no. 2, pp. 435-451, 1994.

[39] Y. Hong, Y. Xu, and J. Huang, "Finite-time control for robot manipulators," Systems \& Control Letters, vol. 46, no. 4, pp. 243-253, 2002.

[40] H. K. Khalil and J. W. Grizzle, Nonlinear Systems, Vol. 3, Prentice-Hall, Upper Saddle River, NJ, USA, 2002.

[41] P. A. Ioannou and J. Sun, Robust Adaptive Control, Vol. 1, PTR Prentice-Hall, Upper Saddle River, NJ, USA, 1996. 